\title{
CONVERGENCE AND CAUCHY STRUCTURES ON LATTICE ORDERED GROUPS
}

\author{
BY
}

RICHARD N. BALL

\begin{abstract}
This paper employs the machinery of convergence and Cauchy structures in the task of obtaining completion results for lattice ordered groups. $\$ 81$ and 2 concern $l$-convergence and $l$-Cauchy structures in general. $\$ 4$ takes up the order convergence structure; the resulting completion is shown to be the DedekindMacNeille completion. $\$ 5$ concerns the polar convergence structure; the corresponding completion has the property of lateral completeness, among others. A simple theory of subset types routinizes the adjoining of suprema in 83. This procedure, nevertheless, is shown to be sufficiently general to prove the existence and uniqueness of both the Dedekind-MacNeille completion in $\$ 4$ and the lateral completion in \$5. A proof of the existence and uniqueness of a proper class of similar completions comes free. The principal new hull obtained by the techniques of adjoining suprema is the type $\mathscr{y}$ hull, strictly larger than the lateral completion in general.
\end{abstract}

As is nearly always true in the field of lattice ordered groups, this research follows a path first trod by Conrad [10] and Holland [14]. The contributions of Papangelou ([19], [20]) and Kenny [15] have also been important. But the novelty of the present approach lies in the systematic application of convergence and Cauchy structure techniques, significantly more powerful than topological and uniformity techniques. These new techniques have been developed recently by Kent and others ([16], [22]). The author owes a more personal debt of gratitude to professors Gary Davis, G. Otis Kenny, and Darrell Kent for many stimulating conversations on these topics.

A collection $\mathscr{F}$ of subsets of $G$ is a filter if $X \supseteq Y \in \mathscr{F}$ implies $X \in \mathcal{F}$ and if $X$, $Y \in \mathscr{F}$ implies $X \cap Y \in \mathscr{F}$. A collection $\mathfrak{T}$ having only the second property is a filter base and generates the filter $\{Y \subseteq G \mid Y \supseteq X \in \mathfrak{N}\}$. We shall not distinguish between filters and filter bases. For example, if $\mathscr{F}$ and $\mathscr{N}$ are both filters, $\mathscr{F} \mathfrak{N}$ is the filter with base $\{F M \mid F \in \mathscr{F}, M \in \mathscr{T}\}$, and similarly for $\mathscr{F}^{-1}$, $\mathscr{F} \vee \mathfrak{T}, \mathscr{F} \wedge g$ and so forth. As another example, if $\mathscr{F}$ is a filter on $G$ and $G \subseteq H$ we shall often think of $\mathscr{F}$ as a filter on $H$. For $g \in G, \dot{g}$ is $\{Y \subseteq G \mid g \in Y\}$, the principal ultrafilter containing $g$.

If $G$ is an $l$-group and $X \subseteq G$ then the convexification of $X$ is $X \sim=\left\{y \mid x_{1} \leqslant y\right.$ $\left.\leqslant x_{2}, x_{i} \in X\right\}$. The lower (upper) bounds of $X$ are $L_{G}(X)\left(U_{G}(X)\right)=\{y \in G \mid y \leqslant x$ $(y \geqslant x)$ all $x \in X\} . L_{G}(X)$ and $U_{G}(X)$ are often written more simply $L(X)$ and $U(X)$. The order closure of $X$, written $\operatorname{ocl}(X)$, is defined inductively. $X_{0}=X$,

Presented to the Society, January 6, 1978; received by the editors December 20, 1978 and, in revised form, June 28, 1979.

AMS (MOS) subject classifications (1970). Primary 06A55, 54A20; Secondary 54F05, 06A45. 
$X_{\alpha+1}=\left\{\bigvee S \mid S \subseteq X_{\alpha}\right\}$ for $\alpha$ even and $X_{\alpha+1}=\left\{\bigwedge S \mid S \subseteq X_{\alpha}\right\}$ for $\alpha$ odd, and $X_{\beta}=\cup\left\{X_{\alpha} \mid \alpha<\beta\right\}$ for limit ordinals $\beta$. ocl $(X)$ is $X_{\eta}$ for some ordinal $\eta$ such that $X_{\eta}=X_{\eta+1}=X_{\eta+2}$.

A good deal of the material in $\S \S 1$ and 2 applies to group convergence and Cauchy structures as well as to $l$-convergence and Cauchy structures. Therefore $G \leqslant H$ is to be understood as meaning that $G$ is a subgroup of $H$ if $G$ and $H$ are merely groups, but that $G$ is an $l$-subgroup of $H$ if both are $l$-groups. Likewise $G<H$ indicates that $G$ is either a proper subgroup or a proper $l$-subgroup of $H$.

1. l-convergence structures. A convergence structure on a set $G$ is obtained by deciding which filter of subsets of $G$ are to converge to which points of $G$ subject only to three very mild restrictions. We designate the convergence of the filter $\mathcal{F}$ on $G$ to the point $g$ in $G$ by $\mathscr{F} \rightarrow g$. Specifically, $(G, \rightarrow)$ is a convergence space if $\dot{g} \rightarrow g$ for all $g \in G$, if $\mathscr{F} \rightarrow g$ and $\mathscr{K} \rightarrow g$ imply $\mathscr{F} \cap \mathfrak{K} \rightarrow g$, and if $\mathscr{F} \supseteq \mathfrak{R} \rightarrow g$ implies $\mathscr{F} \rightarrow g$. The convergence space is Hausdorff if no filter converges to more than one point. $(G, \rightarrow)$ is a convergence group if $G$ is a group and $\rightarrow$ makes the group operations continuous, that is, if $\mathscr{F} \rightarrow f$ and $\mathscr{T} \rightarrow m$ imply $\mathscr{F}^{-1} \rightarrow f^{-1}$ and $\mathscr{F} \mathfrak{T} \rightarrow f m .(G, \rightarrow)$ is an l-convergence group, abbreviated lc-group, if it is a convergence group and if $\rightarrow$ also makes the lattice operations continuous. Every $l$-topology gives rise to an $l$-convergence structure by defining $\mathscr{F} \rightarrow f$ whenever $\mathscr{F}$ is finer than the neighborhood filter of $f$. An $l$-convergence structure which arises in this fashion will be termed topological. As is true for topological l-groups, an l-convergence structure is characterized by the filters which converge to the identity.

Proposition 1.1. Suppose $(G, \rightarrow)$ is an lc-group and that $W$ is the set of filters convergent to 1 . Then

(a) $\mathrm{i} \in \mathscr{W}$,

(b) $\mathscr{F} \supseteq \mathscr{R} \in \mathscr{W}$ implies $\mathscr{F} \in \mathscr{W}$,

(c) $\mathscr{F}, \mathfrak{N} \in \mathscr{W}$ imply $\mathscr{F} \cap \mathfrak{K} \in \mathcal{W}$,

(d) $\mathscr{F} \in \mathcal{W}, g \in G$ imply $g^{-1} \mathscr{F} g \in \mathscr{W}$,

(e) $\mathscr{F} \in \mathcal{Q}$ implies $\mathscr{F}^{2} \in \mathcal{W}$ and $\mathscr{F}^{-1} \in \mathcal{W}$,

(f) $\mathscr{F} \in \mathcal{W}$ and $a \wedge b=1$ imply $\mathscr{F} a \wedge \mathscr{F} b \in \mathscr{W}$.

Conversely, if $W$ is a collection of filters satisfying these conditions then the unique l-convergence structure inducing $\mathcal{W}$ is obtained by declaring $\mathcal{F} \rightarrow g$ if $\mathcal{F}^{-1} \in \mathcal{W}$.

Proof. All the conditions are clearly necessary, and the arguments for the sufficiency of conditions (d) and (e) for the continuity of the group operations are similar to those given for topological groups in Bourbaki [7, p. 222]. We prove only that (f) is sufficient for the continuity of $\wedge$. Assume $\mathscr{F} \rightarrow f$ and $\mathscr{T} \rightarrow m$. Let $\mathscr{F} f^{-1} \cap \mathscr{N} m^{-1}=\mathcal{L} \in \mathscr{W}$ and let $f^{\prime}=f(f \wedge m)^{-1}$ and $m^{\prime}=m(f \wedge m)^{-1}$. Since $f^{\prime} \wedge m^{\prime}=1$ we have $\mathscr{L} f^{\prime} \wedge \mathscr{L} m^{\prime} \in \mathscr{W}$. Therefore $\mathscr{F} \wedge \mathscr{T} \supseteq \mathcal{L} f \wedge \mathscr{L} m=$

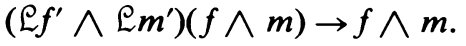

All convergence features of an $l c$-group $G$ can thus be discerned from $\mathscr{W}$. For example, $\rightarrow$ is Hausdorff if and only if $|\cap \mathscr{F}| \leqslant 1$ for all $\mathscr{F} \in \mathcal{W} . \rightarrow$ is topological if and only if QS contains a coarsest filter. $\rightarrow$ is discrete if $i$ is the only flter in 
W. If two convergence structures $\rightarrow$ and $\Rightarrow$ induce $\mho_{1}$ and $\mho_{2}$ then $\rightarrow$ is finer than $\Rightarrow$ (or $\Rightarrow$ is coarser than $\rightarrow$ ) if $\mho_{1} \subseteq \mho_{2}$.

In any convergence space $(G, \rightarrow)$ the closure of a subset $X \subseteq G$, written $\operatorname{cl}_{G}(X)$ or just $\operatorname{cl}(X)$, is $\{g \in G \mid X \in \mathscr{F} \rightarrow g$ for some filter $\mathscr{F}\}$. The closure operator is generally not idempotent, but in case $\operatorname{cl}(X)=X$ we say $X$ is closed and $G-X$ is open. A subset $X \subseteq G$ is dense if $\operatorname{cl}(X)=G$.

For subsets $X$ and $Y$ of an $l$-group $G$ define $X \geqslant Y$ to mean that every $x \in X$ lies above some $y \in Y$ and that every $y \in Y$ lies below some $x \in X$. For filters $\mathscr{F}$ and $\Re$ on $G$ define $\mathscr{F} \preccurlyeq \mathscr{N}$ to mean that for every $F \in \mathcal{F}$ there is an $M \in \mathscr{T}$ with $F \preccurlyeq M$ and also for every $M \in \mathfrak{T}$ there is an $F \in \mathscr{F}$ with $F \preccurlyeq M$.

Proposition 1.2. Suppose $G$ is a sublattice of the Hausdorff lc-group $H$. For $h_{1}$ and $h_{2}$ in $\mathrm{cl}(G)$ the following are equivalent.

(a) $h_{1} \leqslant h_{2}$.

(b) For every $\mathscr{F}_{1} \rightarrow h_{1}$ with $G \in \mathscr{F}_{1}$ there is an $\mathscr{F}_{2} \rightarrow h_{2}$ with $G \in \mathscr{F}_{2}$ and $\mathscr{F}_{1} \preccurlyeq \mathscr{F}_{2}$.

(c) For every $\mathscr{F}_{2} \rightarrow h_{2}$ with $G \in \mathscr{F}_{2}$ there is an $\mathscr{F}_{1} \rightarrow h_{1}$ with $G \in \mathscr{F}_{1}$ and $\mathscr{F}_{1} \preccurlyeq \mathscr{F}_{2}$.

(d) There are filters $\mathscr{F}_{1} \rightarrow h_{1}$ and $\mathscr{F}_{2} \rightarrow h_{2}$ such that $G \in \mathscr{F}_{i}$ and for each $F_{i} \in \mathscr{F}_{i}$ one may find $f_{i} \in F_{i}$ with $f_{1} \leqslant f_{2}$.

Proof. (a) implies (b). Suppose $\mathscr{F}_{1} \rightarrow h_{1}$ and $\mathscr{F} \rightarrow h_{2}$ with $G \in \mathscr{F}_{1} \cap F_{\text {. Let }}$ $\mathscr{F}_{2}=\mathscr{F}_{1} \vee \mathscr{F} \rightarrow h_{2}$. (a) implies (c) is similar while (b) implies (d) and (c) implies (d) are clear. Condition (d) says that filters $\mathscr{F}_{i}$ may be found such that $\mathscr{F}_{i} \rightarrow h_{i}$ and $\mathcal{H}=\left\{Y \subseteq G \mid Y \supseteq\left(F_{1} \wedge F_{2}\right) \cap F_{1}, F_{i} \in \mathscr{F}_{i}\right\}$ is a proper filter. In a Hausdorff lc-group the fact that $\mathcal{K} \rightarrow h_{1} \wedge h_{2}$ and $\mathcal{K} \rightarrow h_{1}$ implies $h_{1}=h_{1} \wedge h_{2}$. The reader is asked to observe that only in (d) implies (a) is the Hausdorff property used.

The next corollary implies that for any $l$-subgroup $G$ of a Hausdorff $l c$-group $H$, $\operatorname{cl}\left(G^{+}\right)=\operatorname{cl}(G)^{+}$.

COROLlary 1.3. Suppose $G$ is an l-subgroup of the Hausdorff lc-group $H$ and that $g \in G$ and $h \in \operatorname{cl}(G)$. Then $g \leqslant h(h \leqslant g)$ if and only if there is a filter $\mathcal{F}$ with $G \in \mathcal{F} \rightarrow h$ such that for every $F \in \mathcal{F}$ there is an $f \in F$ satisfying $f>g(f<g)$.

Proof. In part (b) of Proposition 1.2 let $\mathscr{F}_{1}$ be $\dot{g}$.

Proposition 1.4. Suppose $G$ is a subset of the lc-group $H$.

(a) If $G$ is a subgroup or a sublattice of $H$ then so is $\operatorname{cl}(G)$.

(b) If $C$ is a normal subgroup of the subgroup $G$ of $H$ then $\operatorname{cl}(C)$ is a normal subgroup of $\operatorname{cl}(G)$.

(c) If $C$ is a convex sublattice of the sublattice $G$ of $H$ then $\operatorname{cl}(C)$ is a convex sublattice of $\operatorname{cl}(G)$.

(d) If $C$ is a prime subgroup of the l-subgroup $G$ of $H$ then $\operatorname{cl}(C)$ is a prime subgroup of $\mathrm{cl}(G)$.

Proof. (a) follows directly from the continuity of the operations. To establish (b) suppose that $h \in \operatorname{cl}(G)$ and $m \in \operatorname{cl}(C)$. There must be filters $\mathcal{K}$ and $\mathscr{N}$ such that $G \in \mathcal{H} \rightarrow h$ and $C \in \mathscr{N} \rightarrow m$. Therefore $\mathcal{H}^{-1} \mathscr{N} \mathcal{H} \rightarrow h^{-1} m h$ and is generated 
by sets $A^{-1} B A$ for $G \supseteq A \in \mathcal{H}$ and $C \supseteq B \in \Re$. Each such set must intersect $C$, since for any $a \in A, a^{-1} C a=C$. Therefore $h^{-1} m h \in \operatorname{cl}(C)$. To prove (c), suppose $C$ is a convex sublattice of the sublattice $G$ of $H$ and that $h_{1} \leqslant h \leqslant h_{2}$ for $h_{i} \in \operatorname{cl}(C)$ and $h \in \operatorname{cl}(G)$. There must be filters $\mathscr{F}_{i} \rightarrow h_{i}$ and $\mathscr{F} \rightarrow h$ such that $C \in \mathscr{F}_{i}$ and $G \in \mathscr{F}_{\text {. Let }} \mathscr{F}^{\prime}=\left(\mathscr{F}_{\mathcal{F}} \wedge \mathscr{F}_{2}\right) \vee \mathscr{F}_{1} \rightarrow h$. Note that for an arbitrary $x=\left(g \wedge c_{2}\right) \vee c_{1} \in(G \wedge C) \vee C \in \mathcal{F}^{\prime}$ it is true that $c_{1} \leqslant x \leqslant c_{1} \vee c_{2}$, implying $x \in C$. Therefore $C \in \mathscr{F}^{\prime}$ and $h \in \operatorname{cl}(C)$. To establish (d) suppose that $h_{1}$ and $h_{2}$ are disjoint members of $\operatorname{cl}(G)$ and that $G \in \mathscr{F}_{i} \rightarrow h_{i}$. Let $\mathscr{N}=\left(\mathscr{F}_{1} \wedge \mathscr{F}_{2}\right)^{-1} \rightarrow 1$. For any $F_{i} \in \mathscr{F}_{i}$ and any $f_{i} \in F_{i}$ we have $f_{i}^{\prime}=f_{i}\left(f_{1} \wedge f_{2}\right)^{-1} \in F_{i}^{\prime}=F_{i}\left(F_{1} \wedge F_{2}\right)^{-1}$ $\in \mathscr{F}_{i}^{\prime}=\mathscr{F}_{i} \mathfrak{N} \rightarrow h_{i}$. Since $G \in \mathscr{F}_{i}^{\prime}$ we may assume that $F_{i}^{\prime} \subseteq G$. Since $f_{1}^{\prime} \wedge f_{2}^{\prime}=1$, one of $f_{1}$ and $f_{2}$ must be in $C$. It follows that either $F_{1}^{\prime} \subseteq C$ or $F_{2}^{\prime} \subseteq C$. This is, either $h_{1}$ or $h_{2}$ is in $\operatorname{cl}(C)$.

The next proposition implies that for $G$ an $l$-subgroup of some Hausdorff lc-group, $\operatorname{cl}(G) \subseteq \operatorname{ocl}\left(G^{\sim}\right)$.

Proposition 1.5. If $G$ is a subset of the Hausdorff lc-group $H$ and if $G \in \mathscr{F} \rightarrow h$, then for any $F \in \mathscr{F}, \bigvee(F \wedge h)=\wedge(F \vee h)=h$ in $H$.

Proof. Fix $\mathscr{F}$ and $F$ as above and suppose $x \geqslant F \wedge h$ for some $x \in H$. Then the filter $\mathscr{F} \wedge h$ and $\dot{x}$ satisfy Proposition 1.2(d) (with $G$ there taken to be $H$ here), from which follows $h \leqslant x$.

An $l$-convergence structure $\rightarrow$ is convex if $\mathscr{F} \rightarrow g$ implies $\mathscr{F}^{\sim} \rightarrow g$. Here $\mathscr{F}^{\sim}$ is the filter generated by sets $F \sim$ for $F \in \mathscr{F}$. Given an $l$-convergence structure $\rightarrow$,

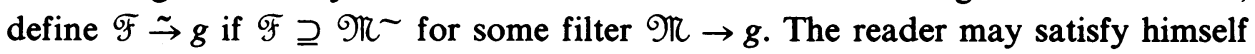
that $\tilde{\rightarrow}$ is the finest convex $l$-convergence structure coarser than $\rightarrow$. As is true in the topological case, there is a rather close connection between $\rightarrow$ and $\tilde{\rightarrow}$; for example, each is Hausdorff only when the other is. For the next proposition the reader should observe that in any $l c$-group $G, \operatorname{cl}\{1\}=\cup\{\cap \mathscr{F} \mid \mathcal{F} \rightarrow 1\}$, an $l$-ideal of $G$ by Proposition 1.4 .

Proposition 1.6. In any lc-group the closures of $\{1\}$ with respect to $\rightarrow$ and $\tilde{\rightarrow}$ coincide.

Proof. Suppose $\mathscr{F} \rightarrow 1$ and $1<x \in \cap \mathscr{F}^{\sim}$. Then $\mathscr{F} \wedge x \rightarrow 1$ and $x \in$ $\cap(\mathscr{F} \wedge x)$ since for all $F \in \mathscr{F}$ there is some $f \in F$ with $x \leqslant f$.

COROLlaRY 1.7. Any l-convergence structure $\rightarrow$ is Hausdorff if and only if $\tilde{\rightarrow}$ is Hausdorff.

It is slightly simpler to check part (f) of Proposition 1.1 in case $\rightarrow$ is a convex group convergence structure.

Proposition 1.8. A convex group convergence structure $\rightarrow$ on an l-group is an l-convergence structure if and only if $\mathscr{F} \rightarrow 1$ implies $\mathscr{F} \wedge \mathscr{F} \rightarrow 1$.

Proof. Suppose $\mathscr{F} \rightarrow 1$ and $a \wedge b=1$. Let $\mathscr{T}=[(\mathscr{F} \wedge \mathscr{F}) \cap(\mathscr{F} \vee \mathscr{F})]^{\sim} \rightarrow 1$. For any $f_{1}, f_{2} \in F \in \mathscr{F}, f_{1} \wedge f_{2}=\left(f_{1} \wedge f_{2}\right) a \wedge\left(f_{1} \wedge f_{2}\right) b<f_{1} a \wedge f_{2} b<$ $\left(f_{1} \vee f_{2}\right) a \wedge\left(f_{1} \vee f_{2}\right) b=f_{1} \vee f_{2}$. That is, $F a \wedge F b \subseteq[(F \wedge F) \cup(F \vee F)]^{\sim} \in$ $\mathscr{T}$. Therefore $\mathscr{F} a \wedge \mathscr{F} b \supseteq \mathscr{T} \rightarrow 1$ and $\mathscr{F} a \wedge \mathscr{F} b \rightarrow 1$. 
An $l$-convergence structure $\rightarrow$ is order closed if $\mathscr{F} \rightarrow g$ implies ocl( $\mathscr{F}) \rightarrow g$. Here $\operatorname{ocl}(\mathscr{F})$ is the filter generated by the $\operatorname{sets} \operatorname{ocl}(F)$ for $F \in \mathscr{F}$. Given an $l$-convergence structure $\rightarrow$, define $\mathscr{F} \Rightarrow g$ if $\mathscr{F} \supseteq \operatorname{ocl}(\mathcal{H})$ for some $\mathscr{H} \rightarrow g$. It is easy to verify that $\Rightarrow$ is the finest order closed $l$-convergence structure coarser than $\rightarrow$. The point of the next proposition is that if $\rightarrow$ is order closed on $G \leqslant H$ then even filters convergent to objects in $\operatorname{cl}(G)$ must have a base of sets order closed in $G$.

Lemma 1.9. Suppose $G$ is an l-subgroup of the lc-group $H$. Then $\rightarrow$ is order closed on $G$ if and only if for every $h \in \operatorname{cl}(G)$ and for every filter $\mathcal{F}$ with $G \in \mathcal{F} \rightarrow h$ it is true that $\operatorname{ocl}_{G}(\mathscr{F}) \rightarrow h$. Here $\operatorname{ocl}_{G}(\mathscr{F})$ is $\left\{Y \subseteq H \mid Y \supseteq \operatorname{ocl}_{G}(F \cap G)\right.$ for some $F \in$ F) .

Proof. The condition certainly implies that $\rightarrow$ is order closed on $G$. Now suppose that $\rightarrow$ is order closed on $G$ and that $G \in \mathscr{F} \rightarrow h$. Let $\mathscr{N}=\operatorname{ocl}_{G}\left(\mathscr{F}^{-1} \mathscr{F}\right)$ $\rightarrow 1$. The generating sets for $\mathscr{T}$ look like $M_{F}=\operatorname{ocl}_{G}\left(F^{-1} F\right)$ for $F \in \mathscr{F}, F \subseteq G$. From each $F \in \mathcal{F}$ choose $f \in F$ and let $\mathcal{L}$ be the filter generated by the sets $f M_{F}$. Since $F \subseteq f M_{F} \subseteq F M_{F}, \mathscr{F} \supseteq \mathcal{L} \supseteq \mathscr{F} \mathscr{N} \rightarrow h$. Since $f M_{F}$ is an order closed subset of $G, \operatorname{ocl}_{G}(\mathscr{F}) \supseteq \mathcal{L} \rightarrow h$.

Proposition 1.10. Suppose $G$ is an l-subgroup of the Hausdorff lc-group $H$. If $\rightarrow$ is order closed on $G$ then $G$ is order dense in $\operatorname{cl}(G)$.

Proof. Suppose $G \in \mathscr{F} \rightarrow h>1$ and let $\mathscr{F}^{\prime}$ be $\operatorname{ocl}_{G}(\mathscr{F} \vee 1)$. The previous proposition asserts $G^{+} \in \mathscr{F}^{\prime} \rightarrow h$. Therefore one may find $F \in \mathscr{F}^{\prime}$ such that $1 \notin F \subseteq G^{+}$and $F$ is order closed. It follows that there is some $g \in G$ with $1<g \leqslant F$ for the only alternative is that $\wedge F=1$, contrary to the assumption that $1 \notin F . g \leqslant h$ by Corollary 1.3 .

An interesting unsettled question is whether, under the hypothesis of Proposition $1.10, \rightarrow$ need be order closed on $\operatorname{cl}(G)$. The next proposition follows directly from Proposition 1.5.

Proposition 1.11. If a convex sublattice $C$ of an l-group $G$ is order closed then it is closed with respect to any Hausdorff l-convergence structure $\rightarrow$.

A convergence structure is regular if $\mathscr{F} \rightarrow g$ implies $\operatorname{cl}(\mathscr{F}) \rightarrow g$. Here $\operatorname{cl}(\mathscr{F})$ is the filter generated by the sets $\operatorname{cl}(F)$ for $F \in \mathscr{F}$. The reader is asked to recall that every topological group convergence structure is regular. The point of the next two results is that convex Hausdorff order closed $l$-convergence structures are also regular.

Proposition 1.12. Suppose $\rightarrow$ is a convex order closed l-convergence structure on the l-group G. Then for any Hausdorff l-convergence structure $\Rightarrow, \mathcal{F} \rightarrow g$ implies $\mathfrak{N} \rightarrow g$ where $\mathfrak{N}$ is the closure of $\mathscr{F}$ with respect to $\Rightarrow$.

Proof. Suppose $\mathscr{F} \rightarrow 1$. Then $\operatorname{ocl}\left(\operatorname{ocl}(\mathscr{F})^{\sim}\right) \rightarrow 1$. The latter is generated by sets $\operatorname{ocl}\left(\operatorname{ocl}(F)^{\sim}\right)$. But $\operatorname{ocl}(F)^{\sim}$ is a convex sublattice, so that $\operatorname{ocl}\left(\operatorname{ocl}(F)^{\sim}\right)$ is an order closed convex sublattice [1]. By Proposition 1.11, $\operatorname{ocl}\left(\operatorname{ocl}(F)^{\sim}\right)$ is closed with respect to $\Rightarrow$. 
COROLlARY 1.13. Every convex Hausdorff order closed l-convergence structure is regular.

A strong semantic link between $G$ and $\operatorname{cl}(G)$ exists. Let $c_{1}, c_{2}, \ldots, c_{m}$ be constants from a convergence group (lc-group) $H$ and let $W_{1}, W_{2}, \ldots, W_{n}$ be words built up from the $c_{i}$ 's and from the variables $v_{1}, v_{2}, \ldots, v_{k}$ using group (and lattice) operations. Let us agree to term any formula obtained by joining the atomic formulas " $W_{i}=1$ " by conjunction or disjunction a disjunctive formula. For any formula $\psi$ having free variables among $v_{1}, v_{2}, \ldots, v_{k}$, abbreviate its universal closure $\forall v_{1}, v_{2}, \ldots, v_{k} \psi$ by $\forall v \psi$. Finally, let $\langle X\rangle$ be the subgroup (l-subgroup) of $H$ generated by $X \subseteq H$.

THEOREM 1.14. Suppose $G$ is a subgroup (l-subgroup) of the Hausdorff convergence group (lc-group) $H$. Let $\psi$ be a disjunctive formula mentioning constants $c_{1}, c_{2}, \ldots, c_{m}$ in $H$. Then $\left\langle G, c_{1}, \ldots, c_{m}\right\rangle \vDash \forall v \psi$ if and only if $\left\langle\operatorname{cl}(G), c_{1}, \ldots, c_{m}\right\rangle$ $\vDash \forall v \psi$.

Proof. Since implication from right to left is clear, suppose $\left\langle\operatorname{cl}(G), c_{1}, \ldots, c_{m}\right\rangle$ $\vDash \forall v \psi$. We may assume $\psi$ to be of the form " $W_{1}=1$ or $W_{2}=1$ or $\ldots$ or $W_{n}=1$." Thus we are assuming we have elements $h_{i} \in\left\langle\operatorname{cl}(G), c_{1}, \ldots, c_{m}\right\rangle$ such that $W\left(h_{1}, \ldots, h_{k}\right) \neq 1$ for $1 \leqslant j \leqslant n$. The continuity of the operations implies that $\left\langle\operatorname{cl}(G), c_{1}, \ldots, c_{m}\right\rangle \subseteq \operatorname{cl}\left\langle G, c_{1}, \ldots, c_{m}\right\rangle$. For each $i$ let $\mathscr{F}_{i}$ be a filter such that $\left\langle G, c_{1}, \ldots, c_{m}\right\rangle \in \mathscr{F}_{i} \rightarrow h_{i}$. Because $W_{j}\left(\mathscr{F}_{1}, \mathscr{F}_{2}, \ldots, \mathscr{F}_{k}\right) \rightarrow W_{j}\left(h_{1}, \ldots, h_{k}\right) \neq 1$, it follows that one may find $F_{i} \in \mathscr{F}_{i}$ such that $1 \notin W_{j}\left(F_{1}, F_{2}, \ldots, F_{k}\right)$ for $1<j<n$. We may assume each $F_{i} \subseteq\left\langle G, c_{1}, \ldots, c_{m}\right\rangle$. Choosing $f_{i} \in F_{i}$, one obtains $W_{j}\left(f_{1}, \ldots, f_{k}\right) \neq 1$ for $1 \leqslant j \leqslant n$. That is, $\left\langle G, c_{1}, \ldots, c_{m}\right\rangle \forall \forall v \psi$.

Suppose $(G, \rightarrow)$ is a convergence group (lc-group) with normal subgroup ( $l$ ideal) $R$ and corresponding natural map $\theta: G \rightarrow G / R$. The quotient convergence structure $\Rightarrow$ on $G / R$ is defined as follows. For $\mathscr{F}$ a filter on $G / R$ and $C$ a coset in $G / R, \mathscr{F} \Rightarrow C$ providing $\mathscr{F} \supseteq \mathfrak{N} \theta$ for some filter $\mathfrak{N}$ on $G$ such that $\mathscr{N} \rightarrow c$ for some $c \in C$. A map $\psi: G \rightarrow H$ between convergence spaces $(G, \rightarrow)$ and $(H, \Rightarrow)$ is continuous if $\mathscr{F} \rightarrow 1$ implies $\mathscr{F} \theta \Rightarrow 1$. It is clear that $\Rightarrow$ is the finest convergence structure on $G / R$ with respect to which $\theta$ is continuous.

Proposition 1.15. Suppose $(G, \rightarrow)$ is a convergence group (lc-group) with normal subgroup (l-ideal) $R$. The quotient convergence structure $\Rightarrow$ is a group convergence structure (l-convergence structure). $\Rightarrow$ is Hausdorff if and only if $M$ is closed. $\Rightarrow$ is discrete if and only if $M$ is open.

Proof. Suppose $\Rightarrow$ is not Hausdorff. There must be some filter $\mathscr{F}$ on $G / R$ such that $\mathscr{F} \Rightarrow 1$ but $1 \neq C \in \cap \mathscr{F}$. We may assume $\mathscr{F}$ to be of the form $\mathscr{T} \theta$ for some $\Re \rightarrow r \in R$. Choose a particular $c \in C$. Then $\Re c^{-1} \rightarrow r c^{-1} \notin R$. But for any $M \in \mathfrak{T}, C \in M \theta$ implies $M c^{-1} \cap R \neq \varnothing$, so that $R$ cannot be closed. If $R$ is not closed then there is a filter $\mathfrak{N}$ such that $R \in \mathfrak{N} \rightarrow m \notin R$. Hence $\mathfrak{T} \theta \rightarrow(m) \theta$ $\neq 1$ but $1 \in \cap \mathfrak{T} \theta$. That is, $\Rightarrow$ is not Hausdorff.

Suppose $\left\{\left(G_{\alpha}, \rightarrow\right) \mid \alpha \in A\right\}$ is a set of convergence spaces, that $G \subseteq \Pi G_{\alpha}$, and that $\pi_{\alpha}: G \rightarrow G_{\alpha}$ is the projection map for each $\alpha$. The product convergence structure 
$\Rightarrow$ on $G$ is defined by declaring $\mathscr{F} \Rightarrow g$ if $\mathscr{F} \pi_{\alpha} \rightarrow g \pi_{\alpha}$ for all $\alpha \in A$. It is clear that the product convergence structure is the coarsest convergence structure on $G$ with respect to which all the $\pi_{\alpha}$ 's are continuous.

Proposition 1.16. Suppose $\left\{\left(G_{\alpha}, \rightarrow\right) \mid \alpha \in A\right\}$ is a set of convergence groups (lc-groups). Then the product convergence structure $\Rightarrow$ is a group convergence structure (l-convergence structure). $\Rightarrow$ is Hausdorff if and only if each $\left(G_{\alpha}, \rightarrow\right)$ is Hausdorff. In the l-group case, $\Rightarrow$ is convex if and only if each $\left(G_{\alpha}, \rightarrow\right)$ is convex. If $G \leqslant \Pi G_{\alpha}$ then $G$ is order closed if and only if each $\left(G_{\alpha}, \rightarrow\right)$ is order closed.

2. l-Cauchy structures. A collection $C$ of filters on a set $G$ is a Cauchy structure if $\dot{g} \in \mathcal{C}$ for every $g \in G$, if $\mathscr{F} \supseteq \mathfrak{T} \in \mathcal{C}$ implies $\mathscr{F} \in \mathcal{C}$, and if $\mathscr{F} \cap \mathfrak{T} \in \mathcal{C}$ whenever $\mathcal{F}$ and $\mathscr{T}$ are in $\mathcal{C}$ and each set of $\mathcal{F}$ intersects each set of $\mathscr{T}$. A natural equivalence relation $\sim$ is imposed on $C$ by declaring that $\mathscr{F} \sim \mathscr{T}$ whenever $\mathscr{F} \cap \mathfrak{K} \in \mathcal{C}$. It is convenient to designate the equivalence classes $\{\mathfrak{T} \mid \mathcal{F} \sim \mathscr{N}\}$ by $[\mathscr{F}]$ and the set of all equivalence classes by $G^{e}$. With each Cauchy structure $C$ is also associated its induced convergence structure $\rightarrow$, obtained by declaring $\mathscr{F} \rightarrow g$ whenever $\mathscr{F} \sim \dot{g}$. Adjectives appropriate to a convergence structure are often applied to the generating Cauchy structure; for example, $\mathcal{C}$ is said to be Hausdorff if $\dot{g}_{1} \cap \dot{g}_{2} \in \mathcal{C}$ implies $g_{1}=g_{2}$.

A Cauchy structure $\mathcal{C}$ is a group Cauchy structure if $\mathscr{F}, \mathfrak{R} \in \mathcal{C}$ imply $\mathscr{F}^{-1}$, $\mathscr{F} \mathfrak{R} \in \mathcal{C}$.

Proposition 2.1. If $\mathcal{C}$ is a group Cauchy structure on group $G$, then for filters $\mathscr{F}$ and $\mathfrak{N}$ in $\mathcal{C}, \mathcal{F} \sim \mathscr{N}$ if and only if $\mathcal{F}^{-1} \mathfrak{T} \rightarrow 1$.

Proof. Let $\mathcal{L}=\mathscr{F} \cap \mathfrak{T}$. Now $\mathcal{L} \in \mathcal{C}$ implies $\mathscr{F}^{-1} \mathfrak{K} \supseteq \mathfrak{L}^{-1} \mathcal{L} \in \mathcal{C}$. Since $1 \in L^{-1} L$ for each $L \in \mathcal{L}$ it follows that $\mathcal{L}^{-1} \mathcal{L} \cap \mathrm{i} \in \mathcal{C}$ and that $\mathscr{F}^{-1} \mathfrak{R} \rightarrow 1$. Conversely, if $\mathscr{F}^{-1} \mathfrak{R} \cap \mathrm{i} \in \mathcal{C}$ then $\mathscr{F} \cap \mathscr{T} \supseteq \mathscr{F}\left(\mathscr{F}^{-1} \mathfrak{R} \cap \mathrm{i}\right) \in \mathcal{C}$.

A group Cauchy structure $C$ is an l-Cauchy structure if $\mathscr{F}, \mathfrak{R} \in \mathcal{C}$ imply $\mathscr{F} \vee \mathscr{T}, \mathscr{F} \wedge \mathscr{K} \in \mathcal{C}$. Define $[\mathcal{F}][\mathscr{T}],[\mathcal{F}]^{-1},[\mathscr{F}] \vee[\mathscr{T}]$ and $[\mathscr{F}] \wedge[\mathscr{T}]$ to be

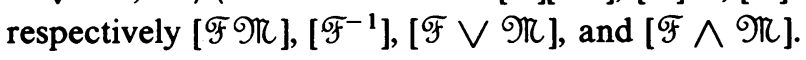

Proposition 2.2. A group Cauchy structure (l-Cauchy structure) $\mathcal{C}$ on group $G$ (l-group $G)$ induces a group convergence structure (l-convergence structure) on $G . G{ }^{\mathcal{E}}$ is a group (l-group) and the map $\psi: G \rightarrow G^{e}$ defined by $(g) \psi=[\dot{g}]$ is a homomorphism (l-homomorphism). $\psi$ is 1-1 if and only if $\mathcal{C}$ is Hausdorff.

$G$ will be considered a subset of $G^{e}$ whenever $\mathcal{C}$ is Hausdorff.

There seems to be no single method of extending the convergence or Cauchy structures on $G$ to $G^{e}$ which is adequate for all our purposes. This is not an obstacle, however, since in the later sections we endow every l-group with a convergence structure $\rightarrow$, and it is necessary only to check whether $\rightarrow$ on $G^{e}$ meshes nicely with $\rightarrow$ on $G$. A reasonable definition of a convergence structure $\Rightarrow$ on $G^{e}$ meshing nicely with the induced convergence structure $\rightarrow$ on $G$ is the following: for $h \in G^{e}$ and $\mathscr{F}$ a filter on $G^{e}$ containing $G, \mathscr{F} \Rightarrow h$ if and only if 
$h=[\mathcal{F}]$. Notice that if $\Rightarrow$ is a convergence structure on $G^{e}$ which meshes nicely with $\rightarrow$ on $G$ then $\Rightarrow$ reduces to $\rightarrow$ on $G$ and $G$ is dense in $\left(G^{e}, \Rightarrow\right)$.

Among those convergence structures on $G^{e}$ which mesh nicely with $\rightarrow$ on $G$, one deserves special mention. For $X \subseteq G$ let $X^{-}=\{[\mathcal{F}] \in G \mathscr{q} X \in \mathscr{F}\}$. For $\mathscr{F}$ a filter on $G$ let $\mathscr{F}^{-}$be the filter on $G^{e}$ generated by the sets $F^{-}$for $F \in \mathcal{F}$. Notice that for $X \subseteq G, X^{-} \cap G=\operatorname{cl}(X)$ with respect to the induced convergence structure on G.

Proposition 2.3. Let $\mathcal{C}$ be a Cauchy structure on $G$ inducing convergence structure $\rightarrow$ on $G$. For $\mathscr{F}$ a filter on $G^{\mathcal{e}}$ define $\mathscr{F} \Rightarrow h \in G^{\mathcal{C}}$ if and only if $\mathscr{F} \supseteq \mathfrak{K}^{-}$for some $\mathfrak{N}$ such that $h=[\mathfrak{R}]$. Then $\Rightarrow$ meshes nicely with $\rightarrow$ if and only if $\mathscr{F} \in \mathcal{C}$ implies $\operatorname{cl}(\mathscr{F}) \in \mathcal{C}$. If $\mathcal{C}$ is a group Cauchy structure (l-Cauchy structure) then $\Rightarrow$ is a group convergence structure (l-convergence structure).

Proposition 2.4. Let $\mathcal{C}$ be a group Cauchy structure inducing $\rightarrow$ on $G$. Then $\rightarrow$ is regular on $G$ if and only if $\mathscr{F} \in \mathcal{C}$ implies $\operatorname{cl}(\mathcal{F}) \in \mathcal{C}$.

Proof. If $\mathscr{F} \in \mathcal{C}$ implies $\mathrm{cl}(\mathscr{F}) \in \mathcal{C}$ then $\rightarrow$ is clearly regular. Suppose $\rightarrow$ is regular on $G$ and that $\mathscr{F} \in \mathcal{C}$. Since $\operatorname{cl}\left(\mathcal{F}^{-1} \mathscr{F}\right) \rightarrow 1$ it follows that $\mathscr{F} \operatorname{cl}\left(\mathscr{F}^{-1} \mathscr{F}\right) \in \mathcal{C}$. For each $F \in \mathscr{F}$ choose $f \in F$. Then $F \subseteq \operatorname{cl}(F) \subseteq \operatorname{cl}\left(f F^{-1} F\right)=f \cdot \operatorname{cl}\left(F^{-1} F\right) \subseteq F$. $\operatorname{cl}\left(F^{-1} F\right)$ so that $\operatorname{cl}(\mathscr{F}) \supseteq \mathscr{F} \operatorname{cl}\left(\mathscr{F}^{-1} \mathscr{F}\right)$ and $\operatorname{cl}(\mathscr{F}) \in \mathcal{C}$.

Because of Proposition 2.4 the formation of $\Rightarrow$ by the definition in Proposition 2.3 will be termed the extension of $\rightarrow$ by regularity.

COROllary 2.5. Suppose $\mathcal{C}$ is an l-Cauchy structure inducing convex Hausdorff order closed l-convergence structure $\rightarrow$ on $G$. Then $\Rightarrow$, the extension of $\rightarrow$ by regularity, is a Hausdorff l-convergence structure on $G^{\mathcal{C}}$ which meshes nicely with $\rightarrow$.

Proof. Under the circumstances $\operatorname{cl}\{1\}$ is an $l$-ideal of $G^{e}$ which, if nontrivial, must have nontrivial intersection with $G$ by Proposition 1.10. But if $1<g \in \operatorname{cl}\{1\}$ for some $g \in G$ then $g \in \cap \mathcal{F}^{-}$for some filter $\mathscr{F}$ on $G$ such that $\mathscr{F} \rightarrow 1$. But then $g \in \cap \operatorname{cl}_{G}(\mathcal{F})$ and $\operatorname{cl}_{G}(\mathscr{F}) \rightarrow 1$, contrary to the assumption that $\rightarrow$ is Hausdorff on $G$.

In case $\mathcal{C}$ and $\mathscr{D}$ are Cauchy structures on $G$ and $H$ respectively, a map $\psi$ : $G \rightarrow H$ is Cauchy continuous providing $\mathscr{F} \in \mathcal{C}$ implies $\mathscr{F} \psi \in \mathscr{D}$. Every Cauchy continuous map is continuous with respect to the induced convergence structures. The next proposition sets forth the canonical extension of a Cauchy continuous homomorphism from $G$ to $G^{e}$.

Proposition 2.6. Suppose $\mathcal{C}$ and $\mathscr{D}$ are group Cauchy structures (l-Cauchy structures) on $G$ and $H$ respectively and that $\psi: G \rightarrow H$ is a Cauchy continuous homomorphism (l-homomorphism). Then the map $\psi^{\wedge}: G^{\mathcal{C}} \rightarrow H^{\mathscr{D}}$ defined by $[\mathcal{F}] \psi=$ $[\mathcal{F} \psi]$ is a homomorphism (l-homomorphism) which extends $\psi$ whenever both $\mathcal{C}$ and $\mathscr{D}$ are Hausdorff. The kernel of $\psi^{\wedge}$ contains $N^{-}$where $N$ is the kernel of $\psi$. Suppose $\rightarrow$ is a group convergence structure (l-convergence structure) on $G^{\mathcal{e}}$ such that $\mathscr{F} \rightarrow[\mathcal{F}]$ for all $\mathcal{F} \in \mathcal{C}$ and suppose that $\Rightarrow$ is a group convergence structure (l-convergence structure) on $H^{\mathscr{D}}$ such that $H \in \mathfrak{N} \Rightarrow m \in H^{\mathscr{D}}$ implies $m=[\mathfrak{T}]$. Suppose also that $\mathcal{C}$ and $\mathscr{D}$ are Hausdorff. If $\theta: G^{e} \rightarrow H^{\mathscr{D}}$ is a homomorphism (l-homomorphism) 
continuous with respect to $\rightarrow$ and $\Rightarrow$ which reduces to $\psi$ on $G$ then $\theta$ is $\psi^{\wedge}$. If $\mathcal{C}$ and (D) are regular then $\psi^{\wedge}$ is the unique extension of $\psi$ which is continuous with respect to the extensions of the convergence structures by regularity.

Proof. Suppose $G, H, \mathcal{C}, \mathscr{D}, \psi, \theta, \rightarrow$ and $\Rightarrow$ are as above and that $h \in G^{\mathcal{C}}$. If $h=[\mathscr{F}]$ then $\mathscr{F} \rightarrow h$ so $\mathscr{F} \theta \Rightarrow(h) \theta$. But since $\theta$ and $\psi$ agree on $G, \mathscr{F} \theta=\mathscr{F} \psi \Rightarrow[\mathscr{F} \psi]$ $=(h) \psi^{\wedge}$. That is, $(h) \theta=[\mathscr{F} \psi]=(h) \psi^{\wedge}$. The rest of the proposition is equally routine.

It is of interest to know when the map $\psi^{\wedge}$ of Proposition 2.6 is one-to-one. One obvious sufficient condition is the following.

Proposition 2.7. Suppose $\mathcal{C}$ and $\mathscr{D}$ are group Cauchy structures (l-Cauchy structures) inducing Hausdorff convergences structures $\rightarrow$ and $\Rightarrow$ on $G$ and $H$ respectively, and suppose $\psi: G \rightarrow H$ is a Cauchy continuous monomorphism (l-monomorphism). If $\psi^{-1}$ is continuous, the $\psi^{\wedge}: G^{\mathcal{C}} \rightarrow H^{\mathscr{D}}$ is one-to-one.

Proof. Consider $1 \neq[\mathscr{F}] \in G^{e}$. Then $\mathscr{F} \psi \neq 1$ since otherwise $\mathscr{F}=\mathscr{F} \psi \psi^{-1} \rightarrow 1$. That is, $[\mathscr{F}] \psi^{\wedge}=[\mathscr{F} \psi] \neq 1$.

COROLlary 2.8. Suppose $C$ is a Hausdorff group Cauchy structure (l-Cauchy structure) on $H$, suppose $G \leqslant H$, and let $\mathscr{D}$ be the restriction of $\mathcal{C}$ to $G$. Then the extension $\psi^{\wedge}$ of the identity map $\psi$ on $G$ is an isomorphism (l-isomorphism) from $G^{D}$ onto $G^{-}$in $H^{e}$.

On account of Corollary $2.8, G^{\mathscr{D}}$ will be considered a subgroup ( $l$-subgroup) of $H^{e}$.

The next proposition provides a more useful sufficient condition for $\psi^{\wedge}$ to be one-to-one.

Proposition 2.9. Suppose $\mathcal{C}$ and $\mathscr{D}$ are group Cauchy structures (l-Cauchy structures) inducing convergence structures $\rightarrow$ and $\Rightarrow$ on $G$ and $H$ respectively, and suppose $\psi: G \rightarrow H$ is a Cauchy continuous homomorphism (l-homomorphism). If $\mathscr{F} \in \mathcal{C}$ implies $(\operatorname{cl}(\mathscr{F} \psi)) \psi^{-1} \in \mathcal{C}$ then $\psi^{\wedge}: G^{\mathcal{C}} \rightarrow H^{\Phi}$ is one-to-one.

Proof. Suppose $1 \neq[\mathscr{F}] \in G^{\mathfrak{e}}$. Then $(\operatorname{cl}(\mathscr{F} \psi)) \psi^{-1} \in[\mathscr{F}] \neq 1$ so there is some $F \in \mathscr{F}$ with $1 \notin(\operatorname{cl}(F \psi)) \psi^{-1}$. Therefore $1 \notin \operatorname{cl}(F \psi), \mathscr{F} \psi \neq 1$ in $H$, and $[\mathscr{F}] \psi^{\wedge}=$ $[\mathscr{F} \psi] \neq 1$.

A slight modification of the proof of Lemma 1.9 proves the following.

LEMma 2.10. Suppose $C$ is an l-Cauchy structure inducing convergence structure $\rightarrow$ on $G$. Then $\rightarrow$ is order closed on $G$ if and only if $\mathscr{F} \in \mathcal{C}$ implies ocl $(\mathscr{F}) \in \mathcal{C} . \rightarrow$ is convex on $G$ if and only if $\mathscr{F} \in \mathcal{C}$ implies $\mathscr{F} \sim \mathcal{C}$.

Proposition 2.11. Suppose $\mathcal{C}$ and $\mathscr{D}$ are l-Cauchy structures inducing convergence structures $\rightarrow$ and $\Rightarrow$ on $G$ and $H$ respectively, and suppose $\psi: G \rightarrow H$ is a Cauchy continuous l-monomorphism. If $\rightarrow$ is convex and order closed and $\Rightarrow$ Hausdorff then $\psi^{\wedge}: G^{\mathcal{C}} \rightarrow H^{\mathscr{D}}$ is one-to-one.

Proof. Propositions 1.11, 2.9, and 2.10. 
Suppose now that $\mathcal{C}$ is a group Cauchy structure ( $l$-Cauchy structure) on $G$ and that $R$ is a normal subgroup ( $l$-ideal) of $G$ with corresponding natural map $\theta$ : $G \rightarrow G / R$. The quotient Cauchy structure $\mathscr{D}$ on $G / R$ is defined by declaring that for a filter $\mathscr{F}$ on $G / R, \mathscr{F} \in \mathscr{D}$ if $\mathscr{F} \supseteq \mathfrak{N} \theta$ for some $\mathscr{N} \in \mathcal{C}$. Clearly $\mathscr{D}$ is the finest Cauchy structure on $G / R$ with respect to which $\theta$ is Cauchy continuous.

Proposition 2.12. Suppose that $\mathcal{C}$ is a group Cauchy structure (l-Cauchy structure) on $G$, that $R$ is a normal subgroup (l-ideal) with natural map $\theta$, and that $\mathscr{D}$ is the quotient Cauchy structure. Then $\mathscr{D}$ is a group Cauchy structure (l-Cauchy structure). The map $\theta^{\wedge}: G^{\mathcal{C}} \rightarrow(G / R)^{\mathscr{D}}$ is an epimorphism (l-epimorphism) with kernel $R^{-}$.

Proof. Suppose $(h) \theta^{\wedge}=1$. By definition there is a filter $\mathscr{F} \in \mathcal{C}$ such that $h=[\mathscr{F}]$ and $\mathscr{F} \theta \cap \mathrm{i} \in \mathscr{D}$, which means $\mathscr{F} \theta \cap \mathrm{i} \supseteq \mathfrak{T} \theta$ for some $\mathscr{N} \in \mathcal{C}$. Let $[\mathfrak{N}]=m \in G^{e}$. For each $M \in \mathscr{N}$ there must exist $F \in \mathscr{F}$ such that $F \boldsymbol{\theta} \cup\{1\} \subseteq$ $M \theta$. The fact that $1 \in M \theta$ implies each $M \in \mathscr{N}$ intersects $R$, so $m \in R^{-}$. The fact that $F \theta \subseteq M \theta$ implies each $F M^{-1}$ also intersects $R$ and $h^{-1} \in R^{-}$. Since $R^{-}$is a subgroup, $h \in R^{-}$.

A version of Proposition 1.15 may be deduced from Proposition 2.12. If $(G, \rightarrow)$ is a Hausdorff convergence group ( $l c$-group) with normal subgroup ( $l$-ideal) $R$ then one obtains a group Cauchy structure ( $l$-Cauchy structure) $\mathcal{C}$ by putting in $\mathcal{C}$ those filters which converge to some point of $G$. The quotient Cauchy structure then induces the quotient convergence structure on $G / R$.

Suppose that for each $\alpha \in A, \bigodot_{\alpha}$ is a Cauchy structure on $G_{\alpha}$, that $G \subseteq \Pi G_{\alpha}$, and that $\pi_{\alpha}: G \rightarrow G_{\alpha}$ is the projection map for each $\alpha$. The product Cauchy structure $\mathcal{C}$ is defined by declaring that $\mathscr{F} \in \mathcal{C}$ if $\mathscr{F} \pi_{\alpha} \in \mathcal{C}_{\alpha}$ for each $\alpha \in A$. $\mathcal{C}$ is clearly the coarsest Cauchy structure on $G$ making each $\pi_{\alpha}$ Cauchy continuous.

Proposition 2.13. Suppose that for each $\alpha \in A, \bigodot_{\alpha}$ is a group Cauchy structure (l-Cauchy structure) on $G_{\alpha}$, that $G$ is a subgroup (l-subgroup) of $\Pi G_{\alpha}$, and that $\pi_{\alpha}$ : $G \rightarrow G_{\alpha}$ is the projection map for each $\alpha$. Then the product Cauchy structure $\mathcal{C}$ is a group Cauchy structure (l-Cauchy structure) inducing the product convergence structure. The map $\pi: G^{e} \rightarrow \Pi G_{\alpha}^{e_{\alpha}}$, defined by declaring $[\mathscr{F}] \pi=x$ where $x \pi_{\alpha}=\left[\mathscr{F} \pi_{\alpha}\right]$, is a monomorphism (l-monomorphism) which is the identity on $G$ whenever $G \subseteq G^{e}$. If $\Sigma G_{\alpha}$ is a subgroup (l-subgroup) of $G$ then $\pi$ is onto.

Proof. Suppose $x \in \Pi G_{\alpha} e_{\alpha}$. For each $\alpha \in A$ choose a filter $\mathscr{F}_{\alpha}$ on $G_{\alpha}$ such that $x \pi_{\alpha}=\left[\mathscr{F}_{\alpha}\right]$. For each finite $B \subseteq A$ and for each choice of $F_{\alpha} \in \mathscr{F}_{\alpha}$ for $\alpha \in B$ let $Y=\cap\left\{F_{\alpha} \pi_{\alpha}^{-1} \mid \alpha \in B\right\}$, a nonempty set providing $\Sigma G_{\alpha}<G$. Let $\mathcal{F}$ be the filter generated by all such $Y$ 's. Then $\mathscr{F} \in \mathcal{C}$ and $[\mathscr{F}] \pi=x$.

Our methods require, for a given group convergence structure $\rightarrow$ on $G$, the production of a group convergence structure $\mathcal{C}$ on $G$ which induces $\rightarrow$. Although in general there are a multitude of such Cauchy structures, a simple and natural definition is the following: $\mathscr{F} \in \mathcal{C}$ if $\mathscr{F}^{-1} \mathscr{F}, \mathscr{F}^{-1} \rightarrow 1$. The reader should satisfy himself that $C$ is a Cauchy structure inducing $\rightarrow$. In the remainder of this paper the Cauchy structure $\mathcal{C}$ will always be related to the convergence structure $\rightarrow$ in 
this way, unless otherwise explicitly specified. If $\rightarrow$ is topological then $\mathcal{C}$ is just the collection of filters Cauchy with respect to the two-sided uniformity of $\rightarrow$. This definition of $\mathcal{C}$ has the advantage that it provides a structure coarser than any group Cauchy structure inducing $\rightarrow$ and thus leads to larger completions in a sense made precise in Proposition 2.15 and Corollary 2.16. It has the disadvantage that it is not always clear that $\mathcal{C}$ is itself a group Cauchy structure.

Proposition 2.14. Suppose $(G, \rightarrow)$ is a convergence group. Then its Cauchy structure $\mathcal{C}$ is a group Cauchy structure if and only if $\mathscr{F}^{-1} \mathscr{F}, \mathscr{F}^{-1}, \mathfrak{N} \rightarrow 1$ imply $\mathscr{F} \mathfrak{N} \mathscr{F}^{-1} \rightarrow 1$ for arbitrary filters $\mathscr{F}$ and $\mathfrak{N}$ on $G$.

Proof. It is clear that $\mathscr{F} \in \mathcal{C}$ implies $\mathscr{F}^{-1} \in \mathcal{C}$. Assuming the condition on $\rightarrow$ holds, consider $\mathscr{F}, \mathfrak{K} \in \mathcal{C}$. Then $(\mathscr{F} \mathscr{N})(\mathscr{F} \mathscr{N})^{-1}=\mathscr{F} \mathscr{N} \mathfrak{K}^{-1} \mathscr{F}^{-1}$. But $\mathfrak{K}^{\mathfrak{K}^{-1}}$ $\rightarrow 1$ since $\mathscr{\Re} \in \mathcal{C}$ and $\mathscr{F} \mathscr{T} \mathfrak{R}^{-1} \mathscr{F}^{-1} \rightarrow 1$ by the condition. A similar argument shows $(\mathscr{F} \mathscr{T})^{-1}(\mathscr{F} \mathscr{T}) \rightarrow 1$. Conversely, assume $C$ to be a group Cauchy structure and consider $\mathscr{F}$ and $\mathfrak{K}$ satisfying $\mathscr{F}^{-1}, \mathscr{F}^{-1} \mathscr{F}, \mathfrak{K} \rightarrow 1$. By definition $\mathscr{F}, \mathfrak{K} \in$ $\mathcal{C}$ so that $\mathscr{F} \mathscr{R} \in \mathcal{C}$. But this says that $(\mathscr{F} \mathscr{T})(\mathscr{F} \mathscr{T})^{-1} \rightarrow 1$. Since it does no harm to assume that $\mathfrak{R} \supseteq \mathrm{i}$, we have $\left(\mathscr{F} \mathscr{T} \mathscr{N}^{-1} \mathscr{F}^{-1}\right) \subseteq \mathscr{F} \mathfrak{K} \mathscr{F}^{-1} \rightarrow 1$.

A group convergence structure $\rightarrow$ satisfying Proposition 2.14 will be termed strongly normal. Every topological group convergence structure is strongly normal [7] and every group convergence structure on an abelian group is clearly strongly normal. A very natural question is to characterize those group convergence structures which are strongly normal. Perhaps they all are.

Proposition 2.15. Suppose $(G, \rightarrow)$ is a convergence group with corresponding Cauchy structure $\mathcal{C}$. If $\mathscr{D}$ is any group Cauchy structure on $G$ inducing $\rightarrow$ then QD $\subseteq \mathcal{C}$. Furthermore, if $\rightarrow$ is strongly normal then $G^{\mathscr{D}}$ can be considered a subgroup of $G^{\mathcal{e}}$ by means of the map $\psi^{\wedge}$ of Proposition 2.8 .

Proof. $\mathscr{F} \in \mathscr{D}$ implies $\mathscr{F}^{-1} \mathscr{F}, \mathscr{F} \mathscr{F}^{-1} \rightarrow 1$ so that $\mathscr{F} \in \mathcal{C}$.

Although it is more easily verified directly, the next proposition is a combination of results 2.8 and 2.15 .

Corollary 2.16. Suppose $(H, \rightarrow)$ is a Hausdorff convergence group and $G$ is a subgroup of $H$ such that $\rightarrow$ is strongly normal on $G$. Let $\mathcal{C}$ be the Cauchy structure corresponding to $\rightarrow$ on $G$. Then the map $\psi: \operatorname{cl}_{H}(G) \rightarrow G^{e}$, defined by $(h) \psi=[\mathscr{F}]$ where $G \in \mathscr{F} \rightarrow h$, is a group monomorphism over $G$.

Our choice of Cauchy structure has the advantage of uniting the concepts of continuity and Cauchy continuity for group homomorphisms.

Proposition 2.17. Suppose $(G, \rightarrow)$ and $(H, \Rightarrow)$ are convergence groups such that $\rightarrow$ is strongly normal. Let $\mathscr{D}$ be any group Cauchy structure inducing $\rightarrow$ and $\mathcal{C}$ the Cauchy structure of $\Rightarrow$.A homomorphism $\theta: G \rightarrow H$ is continuous if and only if it is Cauchy continuous.

Proof. Suppose $\theta$ is continuous and $\mathscr{F} \in \mathcal{C}$. Then $\mathscr{F}^{-1} \mathscr{F} \rightarrow 1$ so $\left(\mathscr{F}^{-1} \mathscr{F}\right) \theta=$ $(\mathscr{F} \theta)^{-1}(\mathscr{F} \theta) \rightarrow 1$. Likewise $(\mathscr{F} \theta)(\mathscr{F} \theta)^{-1} \rightarrow 1$. Therefore $\mathscr{F} \theta \in \mathscr{D}$. 
Our choice of Cauchy structure also makes it slightly easier to verify that a group convergence structure $\Rightarrow$ on $G^{e}$ meshes nicely with $\rightarrow$ on $G$.

Proposition 2.18. Suppose $\rightarrow$ is a strongly normal Hausdorff group convergence structure on $G$ with corresponding Cauchy structure $\mathcal{C}$. A group convergence structure $\Rightarrow$ on $G^{\mathcal{e}}$ meshes nicely with $\rightarrow$ on $G$ if and only if $\mathfrak{N} \Rightarrow[\mathfrak{T}]$ for all $\mathfrak{R} \in \mathcal{C}$ and $\Rightarrow$ reduces to $\rightarrow$ on $G$.

Proof. Suppose $G \in \mathscr{F} \Rightarrow h \in G^{\mathcal{C}}$. Then $h=[\mathcal{H}]$ for some $\mathcal{H} \in \mathcal{C}$. By assumption $\mathcal{H} \Rightarrow h$ so that $\mathcal{L}=\mathscr{F} \cap \mathcal{H} \Rightarrow h$. Therefore $\mathcal{L}^{-1}, \mathfrak{L}^{-1} \mathfrak{L} \Rightarrow 1$. Since $G \in \mathcal{L}^{-1} \mathcal{L}, \mathcal{e} \mathfrak{L}^{-1}$ and since $\Rightarrow$ reduces to $\rightarrow$ on $G$ it follows that $\mathcal{L}^{-1}, \mathfrak{L}^{-1} \mathfrak{L} \rightarrow 1$ and $\mathcal{L} \in \mathcal{C}$. Therefore $h=[\mathcal{H}]=[\mathcal{E}]=[\mathcal{F}]$.

Suppose that $C$ is the Cauchy structure of a strongly normal $l$-convergence structure $\rightarrow$. Even when $\rightarrow$ is topological it is not always true that $\mathscr{F} \wedge \mathscr{R} \in \mathcal{C}$ whenever $\mathscr{F}, \mathfrak{R} \in \mathcal{C}[\mathbf{2 1}]$. However, if $\rightarrow$ is convex then all is well.

Proposition 2.19. The Cauchy structure of a strongly normal convex l-convergence structure $\rightarrow$ is an l-Cauchy structure.

Proof. Assuming $\mathscr{F}, \mathfrak{R} \in \mathcal{C}$ let $\mathcal{L}=\left(\mathscr{F} \mathscr{F}^{-1} \cap \mathscr{T}^{-1} \mathscr{R}^{-1}\right)$ and let $\mathscr{R}=$ $[(\mathcal{L} \vee \mathcal{L}) \cap(\mathcal{L} \wedge \mathcal{L})]^{\sim} \rightarrow 1$. By showing that $(\mathscr{F} \wedge \mathscr{T})(\mathscr{F} \wedge \mathscr{T})^{-1} \supseteq \Re$ and by a similar argument for $(\mathscr{F} \wedge \mathscr{R})^{-1}(\mathscr{F} \wedge \mathscr{R})$ one arrives at the desired conclusion. For any $R \in \mathcal{R}$ there is an $L \in \mathcal{L}$ with $[(L \wedge L) \cap(L \vee L)]^{\sim} \subseteq R$. For this $L$ there must be $F \in \mathscr{F}$ and $M \in \mathscr{N}$ such that $F F^{-1} \cup M M^{-1} \subseteq L$. For $f_{1}, f_{2} \in F$ and $m_{1}, m_{2} \in M$,

$$
\begin{aligned}
\left(f_{1} f_{2}^{-1} \vee f_{1} m_{2}^{-1}\right) \wedge( & \left.m_{1} f_{2}^{-1} \vee m_{1} m_{2}^{-1}\right)=\left(f_{1} \wedge m_{1}\right)\left(f_{2} \wedge m_{2}\right)^{-1} \\
= & \left(f_{1} f_{2}^{-1} \wedge m_{1} f_{2}^{-1}\right) \vee\left(f_{1} m_{2}^{-1} \wedge m_{1} m_{2}^{-1}\right) .
\end{aligned}
$$

Therefore $f_{1} f_{2}^{-1} \wedge m_{1} m_{2}^{-1} \leqslant\left(f_{1} \wedge m_{1}\right)\left(f_{2} \wedge m_{2}\right)^{-1} \leqslant f_{1} f_{2}^{-1} \vee m_{1} m_{2}^{-1}$. Since $f_{1} f_{2}^{-1}, m_{1} m_{2}^{-1} \in L:\left(f_{1} \wedge m_{1}\right)\left(f_{2} \wedge m_{2}\right)^{-1} \in R$. This shows $(F \wedge M)(F \wedge M)^{-1}$ $\subseteq R$ and proves the proposition.

It is worth noting that the $l$-group versions of results $2.15,2.16,2.17$ and 2.18 all hold providing the hypotheses of convexity of $\rightarrow$ is inserted appropriately.

The form of the preceding results most useful for our purposes is summarized in the following corollary.

Corollary 2.20. Suppose $\rightarrow$ is an order closed convex Hausdorff l-convergence structure which is strongly normal on $G$ and suppose $C$ is its l-Cauchy structure. Then $G^{e}$ is an l-group in which $G$ is order dense. The extension of $\rightarrow$ by regularity provides a convex Hausdorff l-convergence structure on $G$ which meshes nicely with $\rightarrow . G$ and $G^{e}$ satisfy the same disjunctive formulas.

Proof. To see that the extension of $\rightarrow$ is convex on $G^{\mathcal{e}}$ consider $\mathscr{F}$ a filter on $G$ such that $\mathscr{F} \rightarrow 1$. Since $\left(\operatorname{ocl}_{G}(\mathscr{F})\right)^{\sim}$ has a base of convex sublattices of $G$ and converges to $1,\left(\left(\operatorname{ocl}_{G}(\mathscr{F})\right)^{\sim}\right)^{-}$has a base of convex sets in $G^{e}$ by Proposition $1.4(\mathrm{c})$. To see that the extension of $\rightarrow$ is Hausdorff on $G^{e}$ recall that $\operatorname{cl}\{1\}$ is an $l$-ideal of $G^{e}$ which, if nontrivial, must intersect $G$ nontrivially. $\operatorname{But}_{G}\{1\}=1$. 
Suppose that a particular convergence, called $x$-convergence and written $\rightarrow$, is defined so that on every group ( $l$-group) $G, \rightarrow$ is (convex) Hausdorff and strongly normal. It is convenient to suppress mention of the Cauchy structure $e$ of $\rightarrow$, writing $G^{x}$ in place of $G^{e}$. We are most interested in the following properties of $\rightarrow$.

$\mathrm{Cl}$ : If $\psi: G \rightarrow H$ is an isomorphism ( $l$-isomorphism) and if $\mathscr{F}$ is a filter on $G$ then $\mathscr{F} \rightarrow 1$ implies $\mathscr{F} \psi \rightarrow 1$.

Property $\mathrm{Cl}$ may be put another way: every isomorphism ( $l$-isomorphism) is continuous. The next property is the only one which does not also apply to convergence groups.

C2: If $G$ is large in $H$ then $\Rightarrow$, the restriction of $\rightarrow$ on $H$ to $G$, is coarser than $\rightarrow$ on $G$. In addition, for any filter $\mathscr{F}$ on $G, \mathscr{F} \rightarrow 1$ implies $\mathfrak{N} \rightarrow 1$ where $\mathfrak{N}$ is the closure of $\mathscr{F}$ with respect to $\Rightarrow$.

The reader should observe that whenever $x$-convergence satisfies $\mathrm{Cl}$ and $\mathrm{C} 2$ every $l$-monomorphism from one $l$-group onto a large $l$-subgroup of another is $x$-continuous.

C3: $\rightarrow$ on $G^{x}$ meshes nicely with $\rightarrow$ on $G$.

A consequence of the next proposition is that $G^{x} \leqslant H^{x}$ whenever $G$ is large in $H$.

Proposition 2.21. Suppose $x$-convergence is a convex Hausdorff strongly normal l-convergence structure defined on every l-group in such a way as to satisfy $\mathrm{C1}, \mathrm{C} 2$ and C3. If $H$ is a l-group large in $H^{x}$ and if $\psi: G \rightarrow H$ is an l-monomorphism such that $G \psi$ is large in $H$ then there is a unique l-isomorphism $\psi^{\wedge}$ mapping $G^{x}$ onto $\operatorname{cl}(G \psi)$ in $H^{x}$ such that $\psi^{\wedge}$ extends $\psi$.

Proof. The existence and uniqueness of $\psi^{\wedge}$ is asserted by Proposition 2.6. The one-to-oneness comes from Proposition 2.9, whose hypotheses are built into C2. Consider an arbitrary $[\mathscr{F}] \in G^{x}$. By C3, $\mathscr{F} \rightarrow[\mathscr{F}]$ so $\mathscr{F} \psi^{\wedge}=\mathscr{F} \psi \rightarrow[\mathscr{F}] \psi^{\wedge}=[\mathscr{F} \psi]$ $\in H^{x}$. Therefore [F] $\psi^{\wedge} \in \operatorname{cl}(G \psi)$ in $H^{x}$. On the other hand if $h \in \operatorname{cl}(G \psi)$ in $H^{x}$ there must be a filter $\mathcal{H}$ on $H^{x}$ with $G \psi \in \mathcal{H} \rightarrow h$. By C3, $h=[\mathcal{H}]$. By Cl and Proposition $2.17, \mathscr{H} \psi^{-1}$ is Cauchy in $G$ so $\left[\mathcal{H} \psi^{-1}\right] \in G^{x}$. Clearly $\left[\mathcal{H} \psi^{-1}\right] \psi^{\wedge}=$ $[\mathcal{H}]=h$, proving $\left(G^{x}\right) \psi^{\wedge}=\operatorname{cl}(G \psi)$ in $H^{x}$.

An l-group $H$ is $x$-complete if $H^{x}=H$. Given $l$-groups $G$ and $H, H$ is said to be an $x$-completion of $G$ if $G$ is large in $H$, if $H$ is $x$-complete, and if $G \leqslant M<H$ implies $M$ is not $x$-complete. If $X \subseteq H$ for $H$ a convergence space, the iterated closure of $X$, written $\operatorname{itcl}_{H}(X)$ or simply itcl $(X)$, is the smallest closed subset of $H$ containing $X$. itcl $(X)$ may also be obtained by iterating the ordinary closure operator transfinitely, taking unions at the limit ordinal stages.

Proposition 2.22. Suppose $x$-convergence satisfies the hypotheses of Proposition 2.21. An l-group $G$ has an $x$-completion if and only if $G$ is large in some $x$-complete l-group $H$, in which case the $x$-completion is $\operatorname{itcl}_{H}(G)$.

Proof. Suppose $G$ is large in the $x$-complete $l$-group $H$. Let $L$ be $\operatorname{itcl}_{H}(G)$. By Proposition 2.21, $L^{x} \leqslant \operatorname{cl}_{H}(L)=L$, so $L$ is $x$-complete. If $G \leqslant M<L$ then $\mathrm{cl}_{H}(M) \neq M$. If $\psi$ is the identity map on $M$ then the extension $\psi^{\wedge}: M^{x} \rightarrow \operatorname{cl}_{H}(M)$ given by Proposition 2.21 is onto, implying $M$ is not $x$-complete. 
Proposition 2.23. Suppose $x$-convergence satisfies the hypotheses of Proposition 2.21. $H$ is an $x$-completion of $G$ if and only if $G$ is large in $H, H$ is $x$-complete, and any l-monomorphism $\psi$ from $G$ onto a large l-subgroup of the $x$-complete l-group $M$ can be uniquely extended to an l-monomorphism $\psi^{\wedge}: H \rightarrow M$.

Proof. Suppose $H$ is an $x$-completion of $G$. The proof of Proposition 2.22 shows that $\operatorname{itcl}_{H}(G)=H$. Let $G=G_{0} \leqslant G_{1} \leqslant \cdots \leqslant G_{\alpha} \leqslant \cdots \leqslant G_{\gamma}=H$ be a sequence of $l$-subgroups of $H$ such that $G_{\alpha+1}=\operatorname{cl}_{H}\left(G_{\alpha}\right)$ and $G_{\alpha}=\cup\left\{G_{\gamma} \mid \gamma<\alpha\right\}$ for limit ordinals $\alpha$. Extend $\psi$ from $G$ to $H$ through the chain of $G_{\alpha}$ 's by repeated use of Proposition 2.21. If $\theta: H \rightarrow M$ is any other $l$-monomorphism which agrees with $\psi$ on $G$ let $\gamma$ be the least ordinal such that $\theta$ and $\psi^{\wedge}$ disagree on $G_{\gamma}$. Then $\gamma=\alpha+1$ for some $\alpha$ and $\theta$ and $\psi^{\wedge}$ agree on $G_{\alpha}$. This situation contradicts the uniqueness clause of Proposition 2.21.

Proposition 2.24. Suppose $x$-convergence satisfies the hypotheses of Proposition 2.21. If an l-group $G$ has an $x$-completion $H$ then it is unique up to an l-isomorphism over $G$. $G$ and $H$ must satisfy the same disjunctive formulas.

The $x$-completion of $G$ will be designated $G^{i x}$, for iterated $x$. Let us write $G \leqslant_{x} H$ to mean that $G$ is a dense subgroup ( $l$-subgroup) with respect to $\rightarrow$ on $H$ and that $\rightarrow$ on $H$ reduces to $\rightarrow$ on $G$.

C4: $G \leqslant H \leqslant M$ and $G \leqslant_{x} M$ imply $G \leqslant_{x} H \leqslant{ }_{x} M$.

Proposition 2.25. Suppose $x$-convergence is a (convex) Hausdorff strongly normal group convergence structure (l-convergence structure) defined on every group (l-group) in such $a$ way as to satisfy $\mathrm{Cl}, \mathrm{C} 3$, and $\mathrm{C} 4$. Then for any groups (l-groups) $G$ and $H$, $G \leqslant_{x} H$ if and only if there is a monomorphism (l-monomorphism) $\psi: H \rightarrow G^{x}$ over $G$.

Proof. If $G \leqslant_{x} H$ then $\psi$ is provided by Corollary 2.16 or its $l$-group analogue. Conversely, if $\psi: H \rightarrow G^{x}$ is a monomorphism ( $l$-monomorphism) which is the identity on $G$ then $G \leqslant H \psi \leqslant G^{x}$. By $\mathrm{C} 3$ and $\mathrm{C} 4, G \leqslant{ }_{x} H \psi$. By $\mathrm{C}_{1}, G<_{x} H$.

Proposition 2.26. Suppose $x$-convergence satisfies the hypotheses of Proposition 2.25. For any group (l-group) $G$ there is an $H$ such that $G<_{x} H$ and such that $H \leqslant M$ and $G \leqslant_{x} M$ imply $H=M$. Every such $H$ is isomorphic (l-isomorphic) to $G^{x}$ over $G$. $G$ and $H$ satisfy the same disjunctive formulas.

The most tractable convergence of all are those which satisfy the converse of $\mathrm{C4}$.

C5: $G \leqslant_{x} H \leqslant_{x} M$ implies $G \leqslant_{x} M$.

Lemma 2.27. Suppose $x$-convergence satisfies the hypotheses of Proposition 2.25 and that $\psi: H \rightarrow G^{x}$ is a monomorphism (l-monomorphism) over $G$. Then $\psi$ is $x$-continuous.

Proof. By Proposition $2.25 G \leqslant_{x} H$. Consider a filter $\mathscr{F}$ on $H$ such that $\mathscr{F} \rightarrow 1$. By $\mathrm{Cl}, \mathscr{F} \psi \rightarrow 1$ in $H \psi$. Now $G<H \psi<G^{x}$, C3 implies $G<{ }_{x} G^{x}$, and $\mathrm{C} 4$ yields $H \psi<_{x} G^{x}$. Therefore $\mathscr{F} \psi \rightarrow 1$ in $G^{x}$. 
Proposition 2.28. Suppose $x$-convergence is a (convex) Hausdorff strongly normal convergence structure (l-convergence structure) defined on every group (l-group) in such $a$ way as to satisfy $\mathrm{C} 1, \mathrm{C} 3, \mathrm{C} 4$ and $\mathrm{C} 5$. Then $G^{x}$ is $x$-complete.

Proof. $G \leqslant_{x} G^{x}<{ }_{x} G^{x x}$ implies $G<{ }_{x} G^{x x}$. By Proposition 2.25 there is a monomorphism ( $l$-monomorphism) $\psi: G^{x x} \rightarrow G^{x}$ over $G$. In fact $\psi$ must be the identity on $G^{x}$. To see this, consider $h \in G^{x}$ and let $\mathscr{F}$ be a filter on $G^{x x}$ such that $G \in \mathscr{F} \rightarrow h$ in $G^{x x}$. By Lemma $2.27, \mathscr{F} \psi \rightarrow(h) \psi$ in $G^{x}$. Since $G^{x}<_{x} G^{x x}, \mathscr{F} \psi \rightarrow$ $(h) \psi$ in $G^{x x}$. Since $\mathscr{F}$ is generated by subsets of $G$ and since $\psi$ is the identity on $G$, $\mathscr{F} \psi=\mathscr{F}$. Since $\rightarrow$ is Hausdorff, $h=(h) \psi$. It follows that $G^{x x}=G^{x}$.

Proposition 2.29. Suppose $x$-convergence is an l-convergence structure which satisfies the hypotheses of Proposition 2.28 and that $G$ is large in $G^{x}$ for each l-group $G$. Then every l-group has an $x$-completion and every such completion is l-isomorphic to $G^{x}$ over $G$.

Proposition 2.30. Suppose $x$-convergence satisfies the hypotheses of Proposition 2.28. Then for any group (l-group) $G$ there is an $H$ such that $G<_{x} H$ and such that $H \leqslant_{x} M$ implies $H=M$. Every such $H$ is isomorphic to $G^{x}$ over $G$.

We close this section by considering the $x$-completions of products and quotients.

C6: For every group ( $l$-group) $G$ and closed normal subgroup ( $l$-ideal) $R, \rightarrow$ is coarser on $G / R$ than the quotient convergence structure $\Rightarrow$. Furthermore, for every filter $\mathscr{F}$ on $G / R, \mathscr{F} \Rightarrow 1$ implies $\mathscr{N} \Rightarrow 1$ where $\mathscr{N}$ is the closure of $\mathscr{F}$ with respect to $\rightarrow$.

The reader should observe that for Hausdorff group convergence structures ( $l$-convergence structures) which satisfy $\mathrm{C6}$, an epimorphism ( $l$-epimorphism) is continuous if and only if its kernel is closed.

Proposition 2.31. Suppose $x$-convergence is a (convex) Hausdorff strongly normal group convergence structure ( $l$-convergence structure) defined on every l-group so that $\mathrm{C} 3$ and $\mathrm{C} 6$ hold. Suppose $R$ is a closed normal subgroup (l-ideal) on $G$ with natural map $\theta: G \rightarrow G / R$ and canonical extension $\theta^{\wedge}: G^{x} \rightarrow(G / R)^{x}$. Then the kernel of $\theta^{\wedge}$ is $\operatorname{cl}(R)$ so that $G^{x} / \operatorname{cl}(R) \leqslant(G / R)^{x}$.

Proof. $\theta^{\wedge}: G^{x} \rightarrow(G / R)^{\mathbf{x}}$ is defined by $[\mathscr{F}] \theta^{\wedge}=[\mathscr{F} \theta]$. In turn, $\theta^{\wedge}$ may be decomposed into $\theta_{1}$ and $\theta_{2}$, where $\theta_{1}: G^{x} \rightarrow(G / R)^{\mathcal{C}}$ and $\theta_{2}:(G / R)^{\mathcal{E}} \rightarrow(G / R)^{x}$, where $\mathcal{C}$ is the quotient Cauchy structure. The kernel of $\theta_{1}$ is $R^{-}$by Proposition 2.12, and $R^{-}=\operatorname{cl}(R)$ since $\mathrm{C} 3$ holds. $\theta_{2}$ is one-to-one by Proposition 2.9. Therefore the kernel of $\theta$ is $\operatorname{cl}(R)$.

C7: If $\left\{G_{\alpha} \mid \alpha \in A\right\}$ is a collection of groups (l-groups) and if $\Sigma G_{\alpha}<G<\Pi G_{\alpha}$ then the product convergence structure $\Rightarrow$ on $G$ is coarser than $\rightarrow$ on $G$. Furthermore, $\mathscr{F} \rightarrow 1$ implies $\mathfrak{N} \rightarrow 1$ where $\mathscr{N}$ is the closure of $\mathscr{F}$ with respect to $\Rightarrow$.

Proposition 2.32. Suppose $x$-convergence is a (convex) Hausdorff strongly normal group convergence structure (l-convergence structure) defined on every group (l-group) in such a way as to satisfy C7. Then for every collection $\left\{G_{\alpha} \mid \alpha \in A\right\}$ of groups 
(l-groups) and for every $G$ with $\Sigma G_{\alpha} \leqslant G \leqslant \Pi G_{\alpha}, G^{x} \leqslant \Pi G_{\alpha}^{x}$. Suppose further that for each $\alpha \in A$ and each filter $\mathscr{F}_{\alpha}$ on $G_{\alpha}$ such that $\mathscr{F}_{\alpha} \rightarrow 1$ it follows that $\mathfrak{N} \rightarrow 1$, where $\mathfrak{N}$ is the filter on $G$ generated by sets of the form $\left\{g \in G \mid g \pi_{\alpha} \in F_{\alpha}\right.$ and $g \pi_{\beta}=1$ for $\left.\beta \neq \alpha\right\}, F_{\alpha} \in \mathscr{F}_{\alpha}, \alpha \in A$. Then $\Sigma G_{\alpha}^{x} \leqslant G^{x}$.

Proof. Proposition 2.9 provides a monomorphism ( $l$-monomorphism) $\theta_{1}: G^{x} \rightarrow$ $G^{e}$ where $\mathcal{C}$ is the product Cauchy structure, while Proposition 2.13 provides an isomorphism ( $l$-isomorphism) $\theta_{2}: G^{e} \rightarrow \Pi G_{\alpha}^{x}$. Now for each $\alpha \in A$ choose $\left[\mathscr{F}_{\alpha}\right] \in$ $G_{\alpha}^{x}$ and define $\mathfrak{N}$ as above. Since $\mathscr{F}_{\alpha} \mathscr{F}_{\alpha}^{-1}, \mathscr{F}_{\alpha}^{-1} \mathscr{F}_{\alpha} \rightarrow 1$ in $G_{\alpha}$ it follows that

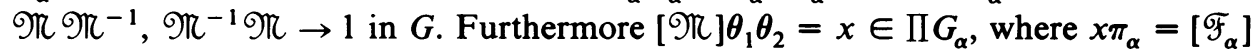
and $x \pi_{\beta}=1$ for $\beta \neq \alpha$.

3. Adjoining suprema. Ever since Dedekind constructed the real numbers by adjoining cuts to the rational numbers there has been an interest in adjoining suprema of particular subsets of a lattice ordered group to obtain a completing structure. The Dedekind-MacNeille completion ([11], [13], [14]) is obtained in exactly this way, adjoining a supremum for each (invertible) cut of an $l$-group $G$. More recently Bernau [4] succeeded in constructing the lateral completion of an arbitrary $l$-group by iteratively adjoining the suprema of pairwise disjoint subsets. The general question becomes: for which types of subsets of $G^{+}$may suprema be adjoined in a consistent way? The Cauchy completion machinery developed in the preceding sections provides an opportunity to approach the adjoining of suprema from a unifying and more general point of view. The lateral completion and the Dedekind-MacNeille completion can then be gotten by parallel applications of the same techniques; the existence and uniqueness of a proper class of previously uninvestigated hulls will be proven as well.

Consider a predicate $\mathcal{X}$ on subsets of $G^{+}$for all $l$-groups $G$. That is, certain subsets of $G^{+}$are of type $\mathcal{X}$, certain are not. We shall refer to $\mathscr{X}$ as a type. The properties of types in which we are interested are the following.

T1: In any $l$-group $G$, for any $g \in G^{+}$and any $X \subseteq G^{+}$of type $\mathcal{X}$ it is true that $1 \vee X g^{-1}$ is also of type $\mathcal{X}$.

T2: Whenever $\psi: G \rightarrow H$ is an $l$-isomorphism and $X$ is of type $\mathcal{X}$ in $G$, it follows that $X \psi$ is of type $\mathcal{X}$ in $H$.

T3: If $G$ is large in $H$ then every subset of type $\mathcal{X}$ in $G$ remains type $\mathcal{X}$ in $H$.

A subset $Y \subseteq G^{+}$is $\mathcal{X}$ order closed if for any subset $X \subseteq Y$ of type $\mathcal{X}$ such that $\bigvee X=y$ it is true that $y \in Y$. An $l$-subgroup $C \leqslant G$ is $\mathscr{X}$ order closed if $C^{+}$is $\mathscr{X}$ order closed. The $\mathcal{X}$ order closure of $Y$ in $G$, written $\mathcal{X o c l}_{G}(Y)$ or simply $\mathscr{X} \operatorname{ocl}(Y)$, is the smallest $\mathcal{X}$ order closed subset of $G^{+}$containing $Y$. An l-homomorphism $\psi$ : $G \rightarrow H$ preserves type $\mathcal{X}$ suprema if $\bigvee X=g$ for $X \subseteq G$ of type $\mathscr{X}$ implies $\bigvee X \psi=g \psi$.

Some examples of types with which the reader may already be familiar are the following: the type of all subsets of $G^{+}$, the type of pairwise disjoint subsets of $G^{+}$, the type of all subsets bounded in cardinality by a particular cardinal, and the type of all invertible cuts (defined in \$4). Several other types will be useful subsequently. Moreover, given two types $\mathcal{X}$ and $\mathcal{Y}$ which have the properties set 
out above, the types formed by joining their predicates by conjunction and disjunction will also have these properties. Thus is obtained, for example, the type of all countable pairwise disjoint subsets.

The form of the next proposition which results from letting $\mathscr{X}$ be the type of all subsets of $G^{+}$is due to Byrd and Lloyd [8].

Proposition 3.1. If $\mathcal{X}$ is a type satisfying $\mathrm{T} 1$ then an l-epimorphism preserves type $\mathfrak{X}$ suprema if and only if its kernel is $\mathcal{X}$ order closed.

Proof. Suppose $\bigvee X=g$ for $X \subseteq G^{+}$of type $\mathcal{X}$ and let $R$ be an $\mathcal{X}$ order closed $l$-ideal of $G$. Clearly $R g \geqslant R x$ for all $x \in X$. Suppose $R y>R x$ for all $x \in X$. That implies $1 \vee x y^{-1}=(y \vee x) y^{-1} \in R$ for all $x \in X$. But $\bigvee\left(1 \vee X y^{-1}\right)=1 \vee(\bigvee X) y^{-1}=1 \vee g y^{-1}$. The hypotheses on $\mathcal{X}$ and $R$ imply $1 \vee g y^{-1} \in R$ so that $R y \geqslant R g$.

An $l$-group $G$ is sup $\mathcal{X}$ complete if every type $\mathcal{X}$ subset of $G$ has a supremum. Given $G$, let us say that $H$ is an $\mathcal{X}$-hull of $G$ providing $G$ is large in $H, H$ is sup $X$ complete, and $G \leqslant M<H$ implies $M$ is not sup $\mathcal{X}$ complete.

Proposition 3.2. Suppose $\mathcal{X}$ is a type satisfying T3. Then an l-group $G$ has an $\mathfrak{X}$ hull if and only if $G$ is large in some sup $X$ complete l-group $H$. In this case $A=\cap\{M \mid G \leqslant M \leqslant H$ and $M$ sup $\mathscr{X}$ complete $\}$ is the unique $\mathscr{X}$ hull of $G$ in $H$.

Proof. Consider an arbitrary $X \subseteq A^{+}$of type $\mathcal{X}$. T3 insures $X$ remains type $\mathcal{X}$ in all $l$-groups $M$ with $A \leqslant M \leqslant H$. Let $\bigvee X=h \in H$. For any sup $\mathcal{X}$ complete $M$ with $A \leqslant M \leqslant H$ there must be some $m \in M$ such that $\bigvee X=m$. But since $M$ is large in $H$, suprema must agree, so $m=h$. Therefore $h \in A$ and clearly $\bigvee X=h$ in $A$. Having proved $A$ sup $\mathfrak{X}$ complete, the proposition follows.

In order to bring the Cauchy constructions to bear on the subject of hulls one must make one more very strong assumption: that $G^{i x}$ is sup $\mathcal{X}$ complete whenever it exists. In the subsequent sections we shall satisfy this requirement by showing that each subset of $G$ of type $\mathcal{X}$ has a supremum in $G^{x}$.

Proposition 3.3. Suppose that $x$-convergence is a convex Hausdorff strongly normal l-convergence structure defined on every l-group in such a way as to satisfy $\mathrm{C} 1, \mathrm{C} 2$ and $\mathrm{C} 3$, and suppose that every l-group $G$ has an $x$-completion $G^{i x}$. Suppose further that $\mathcal{X}$ is a type satisfying $\mathrm{T} 2$ and $\mathrm{T} 3$ and that $G^{i x}$ is sup $\mathcal{X}$ complete. Then for any $\mathfrak{X}$ hull $H$ of $G$ there is an l-monomorphism $\theta: H \rightarrow G^{i x}$ over $G$.

Proof. Since $G$ is large in $H$ and $H$ is large in $H^{i x}, G$ is large in $H^{i x}$. Let $\psi^{\wedge}$ : $G^{i x} \rightarrow H^{i x}$ be the $l$-monomorphism of Proposition 2.23 which extends the identity map on $G$. $H$, which is the unique $\mathcal{X}$ hull of $G$ in $H^{i x}$, must be contained in $G^{i x} \psi^{\wedge}$ since the latter is sup $\mathcal{X}$ complete. Let $\theta$ be the inverse of $\psi^{\wedge}$ restricted to $H$.

Propositions 3.2 and 3.3 immediately yield the desired theorem.

Theorem 3.4. Suppose $x$-convergence and type $\mathcal{X}$ satisfy Proposition 3.3. Then every l-group $G$ has an $\mathcal{X}$ hull which is unique up to an l-isomorphism fixing $G$.

Under the circumstances described in Theorem 3.4 we shall write $G_{\mathfrak{X}}$ for the $\mathscr{X}$ hull of $G$. 
4. Order convergence and the Dedekind-MacNeille completion. Suppose $G$ is an l-group. For $X \subseteq G$ let the lower (upper) bounds of $X$ be designated $L_{G}(X)$ $\left(U_{G}(X)\right)$ or simply $L(X)(U(X))$. It is easy to show that $U L U(X)=U(X)$ and that $L U L(X)=L(X)$ for any $X$. A subset $X \subseteq G$ is a cut if $X=L U(X)$ and $X \neq \varnothing$, $X \neq G$. It should be mentioned that for every $g \in G, L(g)$ is a cut. The purpose of the next proposition is to point out which cuts ought not to have suprema.

LemMA 4.1. If $X$ is any subset of an l-group $G$ such that $\bigvee X=g \in G$, then $X$ is not the union of cosets of any nontrivial convex l-subgroup.

Proof. Suppose $C$ is a nontrivial convex $l$-subgroup of $G$ such that $X=$ $\cup\{C x \mid x \in X\}$. Then for $1<c \in C, c x<g$ implies $x<c^{-1} g<g$ for all $x \in X$, contradicting $\bigvee X=g$.

For cuts $X$ and $Y$ define $X * Y$ to be $L U(X Y)$ and define $X<Y$ if $X \subseteq Y$. The collection of cuts with these operations can be seen to be a lattice ordered semigroup with identity $L(1)$. A cut having an inverse in this semigroup is an invertible cut.

Proposition 4.2. A cut $X$ of an l-group $G$ is invertible if and only if $X$ is neither a union of left cosets nor a union of right cosets of any nontrivial convex l-subgroup of G.

Proof. Suppose $X=\cup\{x C \mid x \in X\}$ for a nontrivial convex l-subgroup $C$ of $G$. If $Y$ is a cut of $G$ such that $Y * X=L(1)$ then $Y X<1$. Fix $1<c \in C$. Then $y x c<1$ implies $y x<c^{-1}$ for all $y \in Y, x \in X$. Therefore $1 \notin L U(Y X)=Y * X$ $=L(1)$, a contradiction. Similarly, the existence of a right inverse for $X$ implies that $X$ cannot be a union of right cosets of any nontrivial convex $l$-subgroup. Now suppose $X$ is a cut which is not invertible. In particular, suppose that $X * L\left(X^{-1}\right)$ $\neq L(1)$ or, equivalently, that $U(X) \cdot(g X)^{-1}$ or $g^{2}>U(X) \cdot X^{-1}$. Therefore $X$ is a union of left cosets of the nontrivial convex $l$-subgroup $\{g \in G|| g \mid<U(X)$. $\left.X^{-1}\right\}$. Similarly, $L\left(X^{-1}\right) * X \neq L(1)$ implies $X$ is a union of right cosets of the nontrivial convex $l$-subgroup $\left\{g \in G|| g \mid<X^{-1} \cdot U(X)\right\}$.

The invertible cuts of $G$ form a lattice ordered group which we will write $G \wedge$. The map $g \rightarrow L(g)$ is an $l$-isomorphism from $G$ into $G^{\wedge}$. Although the terminology has sometimes been applied to the entire semigroup of cuts, we shall term $G^{\wedge}$ the Dedekind-MacNeille completion of $G . G^{\wedge}$ has been well studied for archimedean $G$ [11] and for $G$ totally ordered [14]. Our intention is to capture $G^{\wedge}$ with a Cauchy construction.

A filter $\mathcal{F}$ on an $l$-group $G$ order converges to 1 if $\wedge\left\{t \in G^{+} \mid t^{-1}<F<t\right.$ some $F \in \mathscr{F}\}=1$. For the remainder of this section $\rightarrow$ will mean order convergence while $G^{0}$ and < $<_{0}$ will designate the corresponding completion and density. Order convergence has been studied in the context of lattice theory ([12], [16]); it will soon be seen to have every nice property mentioned in the first three sections. An alternative definition is helpful. A set $T \subseteq G^{+}$is a dual ideal if $t_{1}, t_{2} \in T$ implies $t_{1} \wedge t_{2} \in T$ and if $g \geqslant t \in T$ implies $g \in T$. For any $a, b \in G$ with $a<b$ let $[a, b]_{G}$ or simply $[a, b]$ designate the interval $\{g \in G \mid a<g<b\}$. If $T$ is a dual 
ideal let $\mathfrak{V}_{G}(T)$, or simply $\mathfrak{V}(T)$, designate the filter generated by the intervals $\left[t^{-1}, t\right]_{G}$ for $t \in T$. Then $\mathscr{F} \rightarrow 1$ if and only if $\mathscr{F} \supseteq \mathcal{V}(T)$ for some dual ideal $T$ with $\wedge T=1$.

Proposition 4.3. Order convergence is a convex Hausdorff order closed l-convergence structure on any l-group $G$.

Proof. The convexity and order closure follow from the convexity and order closure of any interval $\left[t^{-1}, t\right]$. The Hausdorff property follows from the requirement that $\wedge T=1$ if $\mathscr{V}(T) \rightarrow 1$. The remaining properties follow from the facts that $\mathscr{V}\left(T_{1}\right) \cap \mathcal{V}\left(T_{2}\right) \supseteq \mathcal{V}\left(T_{1} \cap T_{2}\right), \mathcal{V}(T) \cdot \mathcal{V}(T) \supseteq \mathcal{V}\left(T^{2}\right), g^{-1} \mathcal{V}(T) g=$ $\mathcal{V}\left(g^{-1} T g\right)$, and $\mathcal{V}(T) \wedge \mathcal{V}(T)=\mathcal{V}(T)$. Here we use the fact that if $T_{1}$ and $T_{2}$ are dual ideals with $\wedge T_{1}=\wedge T_{2}=1$, then $T_{1} \cap T_{2}$ satisfies $1=\left(\bigwedge T_{1}\right) \vee\left(\wedge T_{2}\right)=$ $\bigwedge\left(T_{1} \vee T_{2}\right)=\wedge\left(T_{1} \cap T_{2}\right)$. Similarly, $\bigwedge T=1$ implies $1 \cdot 1=(\bigwedge T)(\bigwedge T)=$ $\wedge T^{2}$.

In a sense made precise in Proposition 4.7, order convergence is the finest convex Hausdorff $l$-convergence structure whose (iterated) closure operator agrees with ocl.

LEMMA 4.4. Suppose $\bigvee S=x$ for an upper directed subset $S$ of the l-group $G$ endowed with a convex Hausdorff l-convergence structure $\Rightarrow$. Then $x$ is in the closure of $S$ with respect to $\Rightarrow$ if and only if $\mathfrak{V}\left(x S^{-1}\right) \Rightarrow 1$.

Proof. In this proof and the next only, let cl represent the closure operator of $\Rightarrow$. If $\mathfrak{V}\left(x S^{-1}\right) \Rightarrow 1$ then clearly $1 \in \operatorname{cl}\left(x S^{-1}\right)$ so $x^{-1} \in \operatorname{cl}\left(S^{-1}\right)$ or $x \in \operatorname{cl}(S)$. On the other hand $x \in \operatorname{cl}(S)$ implies $1 \in \operatorname{cl}\left(x S^{-1}\right)$. In this case there must be a filter $\mathscr{F}$ such that $x S^{-1} \in \mathscr{F} \Rightarrow 1$. But then $\mathscr{V}\left(x S^{-1}\right) \supseteq\left(\mathscr{F} \cap \mathscr{F}^{-1}\right)^{\sim} \Rightarrow 1$.

Corollary 4.5. A sublattice $S$ of an l-group $G$ is order closed if and only if $S$ is closed with respect to order convergence.

One of the avowed goals (not fully attained) of $\$ 1$ of [1] was to show that a convex sublattice is order closed if and only if it is closed with respect to every Hausdorff $l$-topology. By relaxing our standards to include convex Hausdorff $l$-convergence structures the result becomes almost trivial.

PROPOSITION 4.6. For a convex sublattice $S$ the following are equivalent.

(a) $S$ is order closed.

(b) $S$ is closed with respect to any convex Hausdorff l-convergence structure.

(c) $S$ is closed with respect to order convergence.

Proof. Proposition 1.11 and Corollary 4.5.

Proposition 4.7. Suppose $\Rightarrow$ is a convex Hausdorff l-convergence structure whose closure operator, written $\mathrm{cl}$, is idempotent on convex sublattices. Then $\Rightarrow$ is coarser than order convergence if and only if $\operatorname{cl}(S)=\operatorname{ocl}(S)$ for all convex sublattices $S$.

Proof. If $\Rightarrow$ is coarser than order closure, then $\operatorname{cl}(S)$ contains the closure of $S$ with respect to order convergence. In fact, since $\mathrm{cl}$ is idempotent and since all mentioned closure operations preserve convex sublattices, $\operatorname{cl}(S)$ contains the 
iterated closure of $S$ with respect to order convergence, namely ocl $(S)$. But $\operatorname{cl}(S) \subseteq \operatorname{ocl}(S)$ by Proposition 1.11. Conversely assume $\operatorname{cl}(S)=\operatorname{ocl}(S)$ for any convex sublattice. In particular, if $T$ is a dual ideal such that $\wedge T=1$ then $1 \in \operatorname{cl}(T)$. That is, $1=\bigvee T^{-1}$ and $1 \in \operatorname{cl}(T)$ which implies by Lemma 4.4 that $\mathfrak{V}(T) \Rightarrow 1$. Since $T$ was arbitrary, $\Rightarrow$ is coarser than order convergence.

An obvious question arises: how many times must the closure operator of order convergence be applied to obtain $\operatorname{ocl}(S)$ for convex sublattice $S$ ? The answer for $S$ a convex $l$-subgroup is of course once. Perhaps there is a complexity measure for convex sublattices here.

Two disparate results from the theory of $l$-groups have ready proofs employing order convergence. The first is a generalization of a result of Bernau [5].

Proposition 4.8. For any l-subgroup $G$ of the l-group $H$ and for any disjunctive formula $\psi, G \vDash \forall v \psi$ if and only if $\operatorname{ocl}(G) \vDash \forall v \psi$.

Proof. Suppose $G=G_{0} \leqslant G_{1} \leqslant G_{2} \leqslant \cdots \leqslant G_{\lambda}=\operatorname{ocl}_{H}(G)$ where for each ordinal $\gamma, G_{\gamma+1}$ is the closure of $G_{\gamma}$ with respect to order convergence on $H$ and where unions are taken at limit ordinals. Theorem 1.14 guarantees that any disjunctive formula $\psi$ holding in $G_{\gamma}$ must hold in $G_{\gamma+1}$. The persistence of $\psi$ through limit stages is an obvious attribute of disjunctive formulas.

Proposition 4.10 is Lemma 3.3 from the classic paper [8] of Byrd and Lloyd. The proof for the topological case of the next lemma given in [1] (Corollary 2.2) carries over to lc-groups. It is due to Madell [18].

LEMMA 4.9. In an lc-group, any convex l-subgroup properly containing a closed prime subgroup is itself both open and closed.

Proposition 4.10. Any convex l-subgroup properly containing an order closed prime subgroup must be prime and order closed.

Proof. If $P$ is an order closed prime and $Q$ is a convex $l$-subgroup containing it then both $P$ and $Q$ are closed with respect to order convergence by Proposition 1.11 and Lemma 4.9. Therefore $Q$ is order closed by Proposition 4.6.

We return now to the subject of the properties of order convergence.

Proposition 4.11. Order convergence is strongly normal.

Proof. Suppose $\mathscr{F}, \mathfrak{K}^{-1} \mathfrak{K}, \mathfrak{K} \mathfrak{K}^{-1} \rightarrow 1$. We may assume $\mathscr{F}=\mathfrak{V}(T)$ and $\Re \Re^{-1}, \Re^{-1} \Re \supseteq \mathcal{R}(S)$ for dual ideals $S$ and $T$ such that $\wedge S=\wedge T=1$. Let $U=\{g \in G \mid M \leqslant g$ for some $M \in \mathscr{T}\}$ and $L=\{g \in G \mid g<M$ for some $M \in \mathfrak{N}\}$. Since $U$ is closed under infima and $L$ is closed under suprema, $U L^{-1}$ generates a dual ideal. It should be observed at this point that $S^{2} \subseteq U L^{-1}$, for if $s \in S$ then there is some $M \in \mathscr{N}$ with $s^{-1}<M M^{-1} \cup M^{-1} M<s$. For a particular $m \in M, s^{-1} m \leqslant M \leqslant s m$, which implies $s m \in U, s^{-1} m \in L$, and $s^{2}=$ $\operatorname{sm}\left(s^{-1} m\right)^{-1} \in U L^{-1}$. The upshot of this observation is that $\wedge U L^{-1}=1$. Our interest lies in the dual ideal $Q$ generated by $U T L^{-1}$. To show that $\wedge Q=1$ consider $g>1$. Since $\wedge U L^{-1}=1$, there must be elements $u \in U$ and $v \in L$ such that $u v^{-1} \ngtr g$. Therefore $u v^{-1} \vee g>u v^{-1}$ or $\left(u v^{-1} \vee g\right) v u^{-1}>1$. Now the fact 
that $\wedge u T u^{-1}=1$ implies the existence of $t \in T$ with $u t u^{-1} \ngtr\left(u v^{-1} \bigvee g\right) v u^{-1}$, that is $u t v^{-1} \ngtr u v^{-1} \vee g$. Since $u t v^{-1} \geqslant u v^{-1}$, it follows that $u t v^{-1} \neq g$. This completes the argument that $\wedge Q=1$. The proof of the proposition is completed by observing that $\mathscr{N} \mathscr{F} \mathfrak{K}^{-1} \supseteq \mathcal{V}(Q)$ since for $u t v^{-1} \in Q$ there are sets $F \in \mathscr{F}$ and $M \in \mathscr{T}$ with $F \subseteq\left[t^{-1}, t\right]$ and $v \leqslant M \leqslant u$, from which it follows that $M F M^{-1} \subseteq\left[\left(u t v^{-1}\right)^{-1}, u t v^{-1}\right]$.

Proposition 4.12. For $G \leqslant H$ the following are equivalent.

(a) For every $h \in H, h=\bigvee L_{G}(h)$.

(b) For every $h \in H^{+}, h=\bigvee L_{G}(h)=\wedge U_{G}(h)$.

(c) For every $h \in H^{+}, 1=\bigwedge\left(U_{G}(h) \cdot L_{G}(h)^{-1}\right)$.

(d) $G \leqslant_{0} H$.

Proof. If (a) holds and $h \in H^{+}$then not only is $h=\bigvee L_{G}(h)$ but also $h^{-1}=\bigvee L_{G}\left(h^{-1}\right)$ so that $h=\left(\bigvee L_{G}\left(h^{-1}\right)\right)^{-1}=\wedge\left(L_{G}\left(h^{-1}\right)\right)^{-1}=\wedge U_{G}(h)$. If (b) holds then $1=h h^{-1}=\left(\bigwedge U_{G}(h) \cdot\left(\bigvee L_{G}(h)\right)^{-1}=\bigwedge\left(U_{G}(h) \cdot L_{G}(h)^{-1}\right)\right.$. (c) clearly implies (b), since if $h>m \geqslant L_{G}(h)$ then $1<h m^{-1} \leqslant U_{G}(h) \cdot L_{G}(h)^{-1}$. To show that (b) implies (a) consider $h \in H$ and let $h^{+}=h \vee 1$ and $h^{-}=h \wedge 1$. Part (c) implies that $h^{+}=\bigvee L_{G}\left(h^{+}\right)$and that $\left(h^{-}\right)^{-1}=\wedge U_{G}\left(\left(h^{-}\right)^{-1}\right)$. Therefore $h=h^{+} h^{-}=\left[\bigvee L_{G}\left(h^{+}\right)\right]\left[\wedge U_{G}\left(\left(h^{-}\right)^{-1}\right)\right]^{-1}=\bigvee\left(L_{G}\left(h^{+}\right) \cdot L_{G}\left(h^{-}\right)\right)$. But $L_{G}\left(h^{+}\right) \cdot L_{G}\left(h^{-}\right) \subseteq L_{G}\left(h^{+} h^{-}\right)=L_{G}(h)$. So $h=\bigvee L_{G}(h)$. Thus far we have the equivalence of (a), (b) and (c). Note that (a) implies that $G$ is order dense in $H$ so that for any dual ideal $T$ of $G, \wedge T=1$ in $G$ if and only if $\wedge T=1$ in $H$. These conditions also imply that $\wedge(Q \cap G)=1$ in $G$ whenever $\wedge Q=1$ in $H$ for any dual ideal $Q$ on $H$. Therefore order convergence on $H$ must reduce to order convergence on $G$. Consider an arbitrary $h \in H^{+}$and let $\mathscr{F}$ be the filter on $H$ generated by sets $[v, u]_{H}$ where $v \in L_{G}(h)$ and $u \in U_{G}(h)$. Then $\mathscr{F}^{-1} \supseteq \mathscr{V}_{H}(Q)$ where $Q=\left\{g \in H \mid g \geqslant u v^{-1}, u \in L_{G}(h), v \in U_{G}(h)\right\}$, a dual ideal. $\mathscr{F} \mathscr{F}^{-1} \rightarrow 1$ since $\wedge Q=1$ by part (c). Hence $\mathscr{F} \supseteq \mathscr{F}^{-1} h \rightarrow h$. Since each set of $\mathscr{F}$ intersects $G, h \in \operatorname{cl}(G)$. Therefore $G$ is dense in $H$ and $G<_{0} H$. Finally, to show (d) implies (c) assume $G \leqslant_{0} H$ and $h \in H^{+}$. Let $\mathcal{F}$ be a filter on $H$ such that $G \in \mathcal{F} \rightarrow h$ and let $T$ be a dual ideal on $G$ such that $\bigwedge T=1$ and $\mathscr{F} \mathscr{F}^{-1} \supseteq \mathcal{V}(T)$. For each $t \in T$ there is an $F \in \mathcal{F}$ with $F F^{-1} \subseteq\left[t^{-1}, t\right]$. Choosing $f \in F \cap G$ yields $t^{-1} f<F<$ tf. By Corollary $1.3, t^{-1} f \leqslant h \leqslant t F$. Since $t^{-1} f \in L_{G}(h)$ and $t f \in U_{G}(h)$, since $t f\left(t^{-1} f\right)^{-1}=t^{2}$, and since $\wedge T^{2}=1$ we can conclude that $1=\wedge\left(U_{G}(h)\right.$. $\left.L_{G}(h)^{-1}\right)$.

Proposition 4.13. Order convergence has properties $\mathrm{C} 1, \mathrm{C} 2, \mathrm{C} 3, \mathrm{C} 4$ and $\mathrm{C} 5$.

Proof. $\mathrm{Cl}$ is obvious while $\mathrm{C} 4$ and $\mathrm{C} 5$ follows from Proposition 4.12 by means of a routine deduction. In order to show $\mathrm{C} 3$ we prove something stronger: that $\rightarrow$ on $G^{0}$ is the extension of $\rightarrow$ on $G$ by regularity. The first step is to show that $\left[t^{-1}, t\right]_{G}^{-}=\left[t^{-1}, t\right]_{G^{0}}$ for any $t \in G^{+}$. For if $h \in\left[t^{-1}, t\right]_{G}^{-}$then $h=[\mathscr{F}]$ for some filter $\mathscr{F}$ on $G$ containing $\left[t^{-1}, t\right]_{G}$. Since for every $F \in \mathscr{F}$ there is some $f \in F$ with $t^{-1} \leqslant f \leqslant t$, it follows from Corollary 1.3 that $t^{-1}<h \leqslant t$. Therefore for every dual ideal $T$ on $G, \mathscr{V}_{G}(T)^{-}=\mathfrak{V}_{G^{0}}(T)$. The second step is to show that $G<{ }_{0} G^{0}$. 
Consider $1<h \in G^{0}$, say $h=[\mathcal{F}]$ for some filter $\mathscr{F}$ on $G$. The argument that (d) implies (c) in Proposition 4.12 shows that $1=\wedge U_{G}(h) L_{G}(h)^{-1}$. From $G<_{0} G^{0}$ it follows that $\wedge(S \cap G)=1$ in $G$ whenever $\wedge S=1$ in $G^{0}$ for a dual ideal $S$ of $G^{0}$. Putting these steps together we get $\mathfrak{V}_{G^{0}}(T) \rightarrow 1$ in $G^{0}$ implies $\mathfrak{V}_{G}(T \cap G) \rightarrow 1$ in $G$ and $\mathfrak{V}_{G^{0}}(T) \supseteq \mathfrak{V}_{G^{0}}(T \cap G)=\mathfrak{V}_{G}(T)$. This completes the proof that order convergence satisfies $\mathrm{C} 3$. To establish $\mathrm{C} 2$ suppose $G$ is large in $H$. Then suprema and infima in $G$ and $H$ agree [6]. Therefore if $T$ is a dual ideal of $G$ then $\wedge T=1$ in $G$ implies $\bigwedge T=1$ in $H$ also. Consequently $\mathscr{V}_{H}(T) \rightarrow 1$. Since $\mathfrak{V}_{G}(T)=\mathfrak{V}_{H}(T)$ $\cap G$, the restriction of order convergence from $H$ to $G$ must be coarser than order convergence on $G$. Finally, since $\rightarrow$ is order closed on $G, \mathscr{F} \rightarrow 1$ implies $\mathscr{R} \rightarrow 1$ where $\mathfrak{T}$ is the closure of $\mathscr{F}$ with respect to the restricted order convergence structure from $H$.

Proposition 4.14. $G^{0}=G^{\wedge}$.

Proof. For any $h \in G^{0}$ we know $h=\bigvee L_{G}(h)=\bigwedge U_{G}(h)$ since $G<_{0} G^{0}$. Observe that $X=L_{G}(h)$ satisfies $U(X)=U_{G}(h)$ and $L U(X)=L_{G}(h)=X$. Similarly $L_{G}\left(h^{-1}\right)$ is a cut of $G$. Since $L_{G}(h) * L_{G}\left(h^{-1}\right)$ is $L_{G}^{(1)}, L_{G}(h) \in G \wedge$. Define the $l$-homomorphism $\psi: G^{0} \rightarrow G^{\wedge}$ by declaring $h \psi=L_{G}(h) . \psi$ is clearly one-to-one since $h=\bigvee L_{G}(h)$. To show $\psi$ is onto consider an arbitrary invertible cut $X$ of $G$. Let $\mathcal{F}$ be the filter on $G$ generated by the intervals $[x, y]_{G}$ where $x \in X, y \in$ $U_{G}(X)$. The argument that (b) implies (d) in Proposition 4.12 can be used to show that $[\mathcal{F}]=h \in G^{0}$. Finally Corollary 1.3 shows that $X<h<U_{G}(X)$, so that $h \psi=X$.

Corollary 4.15. For $G \leqslant H$, any of the conditions of Proposition 4.12 is equivalent to the existence of an l-monomorphism $\psi: H \rightarrow G^{\wedge}$ such that $\psi$ is the identity on $G$.

COROLlARY 4.16. $G$ and $G^{\wedge}$ satisfy the same disjunctive formulas.

An $l$-homomorphism $\psi: G \rightarrow H$ is complete if it preserves all suprema and infima that exist in $G$. Specifically, if $\bigvee S=g$ for some $S \subseteq G$ then $\bigvee S \psi=g \psi$.

Proposition 4.17. For an l-homomorphism $\psi: G \rightarrow H$ the following are equivalent.

(a) $\psi$ is order continuous.

(b) $\psi$ is complete.

(c) The kernel of $\psi$ is order closed and suprema and infima in G $\psi$ and $H$ agree.

Proof. Suppose $\psi$ is order continuous and $\bigvee S=g$ for $S \subseteq g$. We may as well assume that $S$ is a sublattice of $G$, in which case $g S^{-1}$ generates a filter $T$ such that $\wedge T=1$. Therefore $\mathfrak{V}_{G}(T) \psi=\mathfrak{V}_{H}(T \psi) \rightarrow 1$ implying $\wedge T \psi=1$. But then $\wedge(g \psi)(S \psi)^{-1}=1$, or $\bigvee S \psi=g \psi$. That (b) implies (a) is clear. The equivalence of (b) and (c) comes from an application of Proposition 3.1 to the type $\mathcal{X}$ of all subsets of $G^{+}$. 
THEOREM 4.18. For $G \leqslant H$ the following are equivalent.

(a) There is an l-isomorphism $\psi: H \rightarrow G^{\wedge}$ over $G$.

(b) $G \leqslant_{0} H$ and if $G \leqslant_{0} M$ and $H \leqslant M$ then $H=M$.

(c) $G \leqslant_{0} H$ and if $H \leqslant_{0} M$ then $H=M$.

(d) $h=\bigvee L_{G}(h)$ for every $h \in H$, and every invertible cut of $H$ has a supremum.

(e) Given any l-group $M$ and any complete l-homomorphism $\psi: G \rightarrow M$ there is $a$ unique complete l-homomorphism $\psi^{\wedge}: H \rightarrow M^{\wedge}$ such that $\psi^{\wedge}$ extends $\psi$.

$G^{\wedge}$ can also be obtained by the methods of $\S 3$. Define $X \subseteq G$ to be of type $\mathscr{D}$ (for Dedekind) if $L U(X)$ is neither a union of right cosets nor a union of left cosets of any nontrivial convex $l$-subgroup. That is, $X$ is of type $\mathscr{D}$ if and only if $L U(X)$ is an invertible cut.

Lemma 4.19. Suppose $X \subseteq G^{+}$is of type $\mathscr{D}$. Then for any $y \in G, y=\bigvee X$ if and only if $y=\bigvee L U(X)$.

Proof. $y=\bigvee X$ implies $y \in U(X)$ so that $y>L U(X)$. Since $L U(X) \supseteq X$, it follows that $y=\bigvee L U(X)$. On the other hand, $y=\bigvee L U(X)$ implies $y>X$. If $z \geqslant X$ then $z \in U(X)$ so $z>L U(X)$ and $z>y$. Therefore $y=\vee X$.

LemMA 4.20. $X \subseteq G^{+}$is of type $D$ if and only if there is some $1<h \in G^{\wedge}$ such that $\bigvee X=h$.

LEMMA 4.21. If $X \subseteq G$ is a union of the cosets of the convex l-subgroup $C$ then so is $U(X)$ and so is $L(X)$.

Proof. If $X=\cup\{C x \mid x \in X\}$ for a convex l-subgroup $C$, and if $u \in U(X)$ then $u>c^{-1} x$ for all $c \in C$ and $x \in X$. Therefore $c u \in U(X)$. Similarly, $L(X)=$ $\cup\{C v \mid v \in L(X)\}$. The proof for left cosets is analogous.

Proposition 4.22. Type oid satisfies T1, T2 and T3.

Proof. Property T1 is a consequence of Lemma 4.20 and property T2 is obvious. Suppose now that $G$ is large in $H$ and that $X \subseteq G^{+}$is type $\mathscr{D}$ in $G$. If $L U_{H}(X)$ is a union of cosets of the nontrivial convex $l$-subgroup $C$ of $H$ then $Y=L U_{H}(X) \cap G$ is a union of cosets of the nontrivial convex $l$-subgroup $C \cap G$ of $G$. But since $X \subseteq Y \subseteq L U_{G}(X)$, it follows that $L U_{G}(Y)=L U_{G}(X)$. Lemma 4.21 then implies $L U_{G}(X)$ is a union of $C \cap G$ cosets, contrary to assumption.

The next proposition adds one more link to the chain of equivalent conditions in Theorem 4.18.

THEOREM 4.23. Every l-group $G$ has $a \mathscr{D}$ hull. Every such $\mathscr{D}$ hull is l-isomorphic to $G^{\wedge}$ over $G$.

Proof. $G^{\wedge}$ is sup $G^{D}$ complete and $G$ is large in $G^{\wedge}$. The result is thus an application of Theorem 3.4.

We close this section with a consideration of the Dedekind-MacNeille completion of $l$-subgroups, quotients, and sums.

Proposition 4.24. If $G$ is large in $H$ then $G^{\wedge} \subseteq H^{\wedge}$. 
Proof. Proposition 2.16.

Proposition 4.25. Order convergence satisfies property C6.

Proof. Let $R$ be an order closed $l$-ideal of $G$ with natural map $\theta$. Notice that for any dual ideal $T$ of $G, \wedge T=1$ implies $\wedge T \theta=1$ because $R$ is order closed. The quotient convergence structure on $G / R$ is generated by filters $\mathscr{V}(T \theta)$ for dual ideals $T$ on $G$ such that $\bigwedge T=1$. Clearly this structure is finer than the full order convergence structure on $G / R$. The rest of $\mathrm{C} 6$ follows from the fact that $\operatorname{ocl}(\mathcal{V}(T))$ $=\mathfrak{V}(T)$ for any dual ideal $T$, keeping in mind Proposition 1.12.

Proposition 4.26. If $R$ is an order closed l-ideal of $G$ then $\operatorname{cl}(R)$ is order closed in $G^{\wedge}$ and $G^{\wedge} / \operatorname{cl}(R) \leqslant(G / R)^{\wedge}$. If $R$ is large in $G$ then $G^{\wedge} / R^{\wedge} \leqslant(G / R)^{\wedge}$.

Proof. Proposition 2.31 provides $\theta^{\wedge}: G^{\wedge} \rightarrow(G / R)^{\wedge}$ which is order continuous because $\rightarrow$ on $G^{\wedge}$ and $(G / R)^{\wedge}$ is gotten by extending $\rightarrow$ on $G$ and $G / R$ by regularity. Therefore the kernel of $\theta^{\wedge}$, namely $\operatorname{cl}(R)$, must be order closed. The last claim follows directly from Proposition 4.24.

Proposition 4.27. Order convergence satisfies property C7.

Proof. Let $\left\{G_{\alpha} \mid \alpha \in A\right\}$ be a collection of l-groups and suppose $\Sigma G_{\alpha} \leqslant G \leqslant$ $\Pi G_{\alpha}$. Consider a dual ideal $T$ on $G$ such that $\wedge T=1$ in $G$. Since infima agree, $\bigwedge T=1$ in $\Pi G_{\alpha}$ so that $\bigwedge T \pi_{\alpha}=1$ for all $\alpha$. For each $t \in T$ and each finite $B \subseteq A$ let $Y(B, t)=\left\{g \in G \mid\left(t \pi_{\alpha}\right)^{-1} \leqslant g \pi_{\alpha} \leqslant t \pi_{\alpha}\right.$ all $\left.\alpha \in B\right\}$, a nonempty set. Let $\mathscr{F}$ be $\{Y \subseteq G \mid Y \supseteq Y(B, t)$ some finite $B \subseteq A$ and some $t \in T\}$. Then $\mathcal{V}_{G}(T) \supseteq$ $\mathscr{F} \Rightarrow 1$ so $\mathfrak{V}_{G}(T) \Rightarrow 1$. The remainder of $\mathrm{C} 7$ follows from the order closure of order convergence.

Proposition 4.28. For any collection $\left\{G_{\alpha} \mid \alpha \in A\right\}$ of l-groups and for any $G$ such that $\Sigma G_{\alpha} \leqslant G \leqslant \Pi G_{\alpha}$ it follows that $\Sigma G_{\alpha}^{\wedge} \leqslant G^{\wedge} \leqslant \Pi G_{\alpha}^{\wedge}$. Furthermore, $\left(\Sigma G_{\alpha}\right)^{\wedge}=$ $\Sigma G_{\alpha}^{\wedge}$ and $\left(\Pi G_{\alpha}\right)^{\wedge}=\Pi G_{\alpha}^{\wedge}$.

Proof. The first assertion follows from Proposition 2.32. The remaining assertions are left as an exercise for the reader.

Suppose $\mathcal{Y}$ is any type finer than $\mathscr{D}$. That is, suppose every set of type $\mathcal{Y}$ is of type $\mathscr{D}$. If $\mathcal{O}$ has properties $\mathrm{T} 2$ and $\mathrm{T} 3$, then every $l$-group has a type $\mathcal{O}$ hull which is unique up to $l$-isomorphism. For example, let $X \subseteq G^{+}$be of type $\mathcal{Y}$ if $X$ is of type $\mathscr{D}$ and has cardinality less than or equal to $\kappa$, for some fixed cardinal $\kappa$. For another example, let $X \subseteq G^{+}$be of type $\mathcal{Y}$ if $X$ is of type $\mathscr{D}$ and $X$ is pairwise disjoint.

It is tempting to conclude this section with a description of $G^{\wedge}$ in case $G$ is completely distributive. Such descriptive theorems follow readily from the fact that completely distributive $l$-groups have an abundance of order closed prime subgroups and from Propositions 4.24, 4.26, and 4.28. These descriptive theorems, however, are no sharper than the analogous representations of $G^{\alpha}$, the completion of $G$ with respect to its $\alpha$-convergence structure, which contains $G^{\wedge}$. These results will be part of a forthcoming study of $\alpha$-convergence. 
5. Polar convergence and completion. A filter $\mathscr{F}$ on an $l$-group $G$ p-converges to 1 if $\cap\left\{F^{\perp \perp} \mid F \in \mathscr{F}\right\}=1$. The resulting convergence structure is the polar convergence structure; the convergence structure, its corresponding completion, and its density relation will be designated $\rightarrow, G^{p}$, and $\leqslant_{p}$, respectively. The fact that the polars of an l-group form a complete Boolean algebra is of repeated usefulness in this section. If $\mathscr{P}$ is a collection of polars, $\bigvee \mathscr{P}$ and $\cap \mathcal{P}$ designate the supremum and infimum of $\mathcal{P}$ in the Boolean algebra of polars.

Proposition 5.1. Polar convergence is a convex Hausdorff order closed l-convergence structure on any l-group $G$.

Proof. Suppose $\mathscr{F} \rightarrow 1$ and $\mathscr{R} \rightarrow 1$ and let $\mathcal{E}=\left\{Y \subseteq G \mid Y \supseteq(F \cup M)^{\perp \perp}\right.$, $F \in \mathscr{F}, M \in \mathscr{T}\}$. In the complete Boolean algebra of polars, $1=\cap F^{\perp \perp} \bigvee$ $\cap M^{\perp \perp}=\cap\left(F^{\perp \perp} \vee M^{\perp \perp}\right)=\cap \mathcal{L}$. Since $\mathscr{F} \cap \mathfrak{T} \supseteq \mathcal{L}$, part (c) of Proposition 1.1 holds. All the other parts of Proposition 1.1 together with the convexity and order closedness of $\rightarrow$ are direct results of the fact that $F^{\perp \perp}$ is an order closed convex $l$-subgroup for any set $F \subseteq G$.

\section{Proposition 5.2. Polar convergence is strongly normal.}

Proof. Suppose $\mathscr{F}$ and $\mathscr{T}$ are filters on $G$ such that $\mathscr{F}_{\mathcal{F}}^{-1}, \mathscr{T} \rightarrow 1$ and consider an arbitrary $1<g \in G$. Choose $F \in \mathcal{F}$ such that $g \notin\left(F F^{-1}\right)^{\perp \perp}$, say $1<x \leqslant g$ and $x \in\left(F F^{-1}\right)^{\perp}$. Choose a specific $f \in F$ and $M \in \Re$ such that $1<f^{-1} x f \notin M^{\perp \perp}$ or $x \notin\left(f M f^{-1}\right)^{\perp \perp}$, say $1<z \leqslant x$ with $z \in\left(f M f^{-1}\right)^{\perp}$. But $x \wedge\left|f_{1} f_{2}^{-1}\right|=1$ implies $z \wedge\left|f_{1} f_{2}^{-1}\right|=1$ so that $z$ and $f_{1} f_{2}^{-1}$ commute for all $f_{1}$, $f_{2} \in F$. Therefore $f_{2}^{-1} z f_{2}=f_{1}^{-1} f_{1} f_{2}^{-1} z f_{2} f_{1}^{-1} f_{1}=f_{1}^{-1} z f_{1}$ for all $f_{1}, f_{2} \in F$. From this one may conclude that $z \in\left(f_{1} M f_{1}^{-1}\right)^{\perp}$ for any $f_{1} \in F$. But since $\cap\left\{\left(f_{1} M f_{1}^{-1}\right)^{\perp} \mid f_{1}\right.$ $\in F\}=\left(F M F^{-1}\right)^{\perp}$, it follows at last that $g \notin\left(F M F^{-1}\right)^{\perp \perp}$. Therefore $\mathscr{F} \mathscr{R} \mathscr{F}^{-1}$ $\rightarrow 1$.

The next proposition is a folk theorem, proved here for completeness.

Proposition 5.3. Suppose $G \leqslant H$. The polars of $G$ and $H$ are in one-to-one correspondence via the intersection map if and only if every nontrivial polar of $H$ has nontrivial intersection with $G$.

Proof. Suppose $G \leqslant H$ and every nontrivial polar of $H$ has nontrivial intersection with $G$. Given a set $X \subseteq G$ let $X^{\perp}$ designate $\{h \in H|| h|\wedge| x \mid=1$ all $x \in X\}$ and, in this proof only, let $X^{\prime}$ designate $X^{\perp} \cap G$. For polars $P$ and $Q$ of $H, P \neq Q$ implies either $P \cap Q^{\perp}$ or $Q \cap P^{\perp}$ is a nontrivial polar and hence intersects $G$ nontrivially. It follows that $P \cap G \neq Q \cap G$, so that the intersection map is one-to-one. If $P$ is a polar of $H$ then $P \cap G \subseteq(P \cap G)^{\perp \perp} \subseteq P$ so $(P \cap G)^{\perp \perp} \cap G=P \cap G$. Therefore $(P \cap G)^{\perp \perp}=P$. It remains only to show that $P \cap G$ is a polar of $G$. To that end it is enough to show $(P \cap G)^{\prime}=P^{\perp} \cap G$. Since $(P \cap G)^{\prime} \supseteq P^{\perp} \cap G$ is clear, consider $1<x \in(P \cap G)^{\prime}$. If $x \notin P^{\perp}$ then there must be some $1<p \in P$ with $p \leqslant x$. But since $(P \cap G)^{\prime} \subseteq(P \cap G)^{\perp}$, $x \in(P \cap G)^{\prime}$, contradicting $(P \cap G)^{\perp \perp}=P$. 
COROLlary 5.4. If $G \leqslant H$ and every nontrivial polar of $H$ has nontrivial intersection with $G$ then polar convergence on $H$ reduces to polar convergence on $G$.

Proposition 5.5. $\rightarrow$ on $G^{p}$ meshes nicely with $\rightarrow$ on $G$.

Proof. The previous corollary shows that $\rightarrow$ on $G^{p}$ reduces to $\rightarrow$ on $G$. By Proposition 2.18 it remains only to show that $\mathscr{F} \rightarrow[\mathscr{F}] \in G^{p}$. To that end consider a Cauchy filter $\mathscr{F}$ on $G$ and call $[\mathscr{F}]=h \in G^{p}$. For each $F \in \mathscr{F}$ let $Q_{F}$ be $\left(F F^{-1}\right)^{\perp} \cap G$. For fixed $F \in \mathscr{F}$ consider an arbitrary $1<x \in Q_{F}$. For any $f \in F$, $\left|f h^{-1}\right| \wedge x=\left[\left|f \mathscr{F}^{-1}\right| \wedge x\right]$, and $\left|f \mathscr{F}^{-1}\right| \wedge x$ is generated by sets of the form $\left|f F_{0}^{-1}\right| \wedge x$ for $F_{0} \in \mathscr{F}$. But for any $f_{0} \in F \cap F_{0},\left|f f_{0}^{-1}\right| \wedge x=1$. Therefore $\left|f h^{-1}\right| \wedge x=1$ for every $x \in Q_{F}$ and $f \in F$, which is to say $F h^{-1} \subseteq Q_{F}^{\perp}=$ $\left(F F^{-1}\right)^{\perp \perp}$. Since $\mathscr{F} \mathcal{F}^{-1} \rightarrow 1, \cap\left\{\left(F F^{-1}\right)^{\perp \perp} \mid F \in \mathscr{F}\right\}=1$ so that $\cap\left\{\left(F h^{-1}\right)^{\perp \perp} \mid F\right.$ $\in \mathscr{F}\}=1$. That is, $\mathscr{F} h^{-1} \rightarrow 1$ and $\mathscr{F} \rightarrow h$.

Proposition 5.6. Polar convergence satisfies $\mathrm{C} 1, \mathrm{C} 2$ and $\mathrm{C} 3$.

LEMMA 5.7. For $G \leqslant H$ the following are equivalent.

(a) $G \leqslant p H$.

(b) Every nontrivial polar of $\boldsymbol{H}$ has nontrivial intersection with $G$. Furthermore, for every $h \in H^{+}$there is a filter $\mathcal{F}$ of polars of $H$ such that $\cap \mathcal{F}=1$ and for every $P \in \mathcal{F}$ there is some $\mathrm{g} \in G$ with $\mathrm{hg}^{-1} \in P$.

(c) There is an l-monomorphism $\psi: H \rightarrow G^{p}$ over $G$.

Proof. Assuming (a) holds, we know $G$ is order dense in $G^{x}$ and hence in $H$, so the first part of (b) must hold. Consider $1<h \in H$, let $\mathscr{N}$ be a filter such that $G \in \mathscr{T} \rightarrow h$ and let $\mathscr{F}=\left\{\left(M h^{-1}\right)^{\perp \perp} \mid M \in \mathscr{T}\right\}$. Since $\mathscr{N} h^{-1} \rightarrow 1, \cap \mathscr{F}=1$. For each $\left(M h^{-1}\right)^{\perp \perp} \in \mathscr{F}$ there is a $g \in G \cap M$ such that $\left(h g^{-1}\right)=\left(g h^{-1}\right)^{\perp \perp}$. Now suppose (b) holds. By the previous corollary, polar convergence on $H$ reduces to polar convergence on $G$. Consider an arbitrary $h \in H^{+}$and let $\mathscr{F}$ be a filter of polars on $H$ such that for each $P \in \mathcal{F}, M(P)=\left\{g \in G \mid h g^{-1} \in P\right\} \neq \varnothing$. Notice that $M\left(P_{1}\right) \cap M\left(P_{2}\right)=M\left(P_{1} \cap P_{2}\right)$, so that the $M(P)$ 's generate a filter $\mathfrak{N}$ containing $G$. Since $\mathscr{T} h^{-1}$ is generated by sets $M(P) h^{-1} \subseteq P$ and since $\cap \mathscr{F}=1$, $\mathfrak{N} h^{-1} \rightarrow 1$. Therefore $\mathfrak{N} \rightarrow h$. Thus far we have proved the equivalence of (a) and (b). The equivalence of (a) and (c) is Proposition 2.25, whose hypotheses require the next corollary. Fortunately, the corollary depends only on the equivalence of (a) and (b).

Corollary 5.8. Polar convergence satisfies C4.

An interesting open question is whether the extension by regularity of polar convergence on $G$ agrees with polar convergence on $G^{p}$. A related question is whether $\operatorname{cl}(P)$ coincides with $P^{\perp \perp}$ in $G^{p}$, where $P$ is a polar of $G$. It is clear that $\operatorname{cl}(P) \subseteq P^{\perp \perp}$ since the latter is order closed and hence closed. The point of the following example is that $\operatorname{cl}(P)$ need not be all of $P^{\perp \perp}$.

Let $\Gamma$ be the set $R \times\{0,1\}$ partially ordered by declaring $(r, a)<(s, b)$ if and only if $r=s$ and $a \leqslant b$. Let $H$ be $V(\Gamma, R)$ (see [9]) and let $G$ be those members of $H$ continuous on the top copy of $R$. The polars of $G$ and $H$ are in one-to-one 
correspondence with the subsets of $R$ and $G^{p}=H$. Let $P$ be $\{g \in G \mid(r, a) g=0$ for all $r$ rational $\}$, a polar of $G$. Then $\operatorname{cl}(P)=\{h \in H \mid(r, a) h=0$ for $r$ rational or $a=1\}$ while $P^{\perp \perp}=\{h \in H \mid(r, a) h=0$ for $r$ rational $\}$. Even in this example, however, the extension by regularity of polar convergence on $G$ agrees with polar convergence on $G^{p}$.

Suppose $\psi: G \rightarrow H$ is a $p$-continuous $l$-homomorphism. An open question central to the study of $G^{p}$ is whether the canonical extension $\psi^{\wedge}: G^{p} \rightarrow H^{p}$ is also $p$-continuous. Under stronger hypotheses $\psi^{\wedge}$ must be $p$-continuous. Let us term an l-homomorphism strongly $p$-continuous if for any filter of polars $\mathscr{F}$ on $G$, $\cap\left\{(P \psi)^{\perp \perp} \mid P \in \mathscr{F}\right\}=(\cap \mathscr{F}) \psi^{\perp \perp}$. The restriction of this condition to filters $\mathscr{F}$ on $G$ such that $\cap \mathcal{F}=1$ yields the apparently weaker definition of $p$-continuity. The question becomes: is every $p$-continuous $l$-homomorphism strongly $p$-continuous? Notice that every $l$-monomorphism $\psi$ from $G$ onto a large $l$-subgroup of $H$ is strongly $p$-continuous.

Lemma 5.9. Suppose that $\psi: G \rightarrow H$ is a strongly p-continuous l-homomorphism and that $P$ is a polar of $G$. Then in $G^{p}$ and $H^{p},\left(P^{\perp \perp}\right) \psi^{\wedge} \subseteq(P \psi)^{\perp \perp}$.

Proof. Consider $1<x \in P^{\perp \perp}$ in $G^{p}$ and let $\mathcal{F}$ be a filter of polars on $G$ satisfying part (b) of Lemma 5.7. For each $Q \in \mathscr{F}$ let $M(Q)=\left\{g \in G \mid x g^{-1} \in\right.$ $Q$ \} and let $\mathfrak{N}$ be the filter generated by the $M(Q)$ 's. Observe that for $Q \in \mathcal{F}$, $g \in M(Q)$ implies $g=\left(x g^{-1}\right)^{-1} x \in(Q \vee P)^{\perp \perp}$ in $G^{p}$. Since $(Q \vee P)^{\perp \perp} \cap G=$ $Q \vee P$, it follows that $Q \vee P \in \Re$ and that $x \in \operatorname{cl}(Q \vee P)$. Therefore $\Re \psi \rightarrow$ $x \psi^{\wedge}$ in $H^{p}$ and $x \psi^{\wedge} \in \operatorname{cl}((P \vee Q) \psi) \subseteq(P \vee Q) \psi^{\perp \perp}$, an order closed convex l-subgroup. Since $\cap \mathscr{F}=1$ it follows that $\cap\{P \vee Q \mid Q \in \mathscr{F}\}=P$. Since $\psi$ is strongly $p$-continuous, $\cap\left\{(P \vee Q) \psi^{\perp \perp} \mid Q \in \mathscr{F}\right\}=P \psi^{\perp \perp}$. Therefore $x \in P \psi^{\perp \perp}$.

Proposition 5.10. Any strongly p-continuous l-homomorphism $\psi: G \rightarrow H$ has $a$ unique strongly p-continuous l-homomorphism $\psi^{\wedge}: G^{p} \rightarrow H^{p}$ extending $\psi$.

Proof. Suppose $\mathscr{F}$ is a filter of polars on $G$ with intersection $Q$. By the previous lemma, $\left(P \psi^{\wedge}\right)^{\perp \perp}=(P \cap G) \psi^{\perp \perp}$ for all $P \in \mathcal{F}$. Therefore

$$
\bigcap\left(P \psi^{\wedge}\right)^{\perp \perp}=\bigcap(P \cap G) \psi^{\perp \perp}=(Q \cap G) \psi^{\perp \perp}=\left(Q \psi^{\wedge}\right)^{\perp \perp}
$$

THEOREM 5.11. For any l-group $G$ there is an l-group $H$ such that $G \leqslant_{p} H$ and such that $H \leqslant K$ and $G \leqslant_{p} K$ imply $H=K$. Every such $H$ is l-isomorphic to $G^{p}$ over $G$. Any strongly p-continuous l-homomorphism $\psi: G \rightarrow K$ has a unique strongly $p$-continuous extension $\psi^{\wedge}: H \rightarrow K^{p} . G$ and $H$ satisfy the same disjunctive formulas.

The next proposition requires the following definition: $G \preccurlyeq H$ means $G$ is order dense in $H$ and $\cap\left\{\left(h g^{-1}\right)^{\perp \perp} \mid g \in G\right\}=1$ for all $h \in H^{+}$. This condition is implied by $G \leqslant_{p} H$ but is not equivalent to it. For example, if $G$ is any representable $l$-group and $H$ is its orthocompletion then $G \preccurlyeq H$, though it is not generally true that $G \leqslant_{p} H$.

In the next proposition $|A|$ symbolizes the cardinality of $A$ and ${ }^{A} B$ symbolizes the set of maps from $A$ to $B$. 
LemMA 5.12. Suppose $\mathcal{P}$ is the collection of polars of an l-group $G$. Then $G \preccurlyeq H$ implies $|H| \leqslant\left|{ }^{9} G\right|$.

Proof. Well order $G$ and let * be some object not in $G$. With each $h \in H^{+}$ associate the map $f_{h}: \mathscr{P} \rightarrow G \cup\{*\}$ defined by declaring $(P) f_{h}$ to be the first $g$ such that $h g^{-1} \in P$. If no such $g$ exists, then $(P) f_{h}$ is defined to be $*$. If $h$ and $k$ are different members of $H^{+}$let $Q$ be any polar such that $h k^{-1} \notin Q$ and $(Q) f_{h}=g \neq$ *. Such a $Q$ is certain to exist since $\cap\left\{P \mid(P) f_{h} \neq *\right\}=1$ because $\cap\left\{\left(h g^{-1}\right)^{\perp \perp} \mid g\right.$ $\in G\}=1$. If $(Q) f_{k}=g$ then $h k^{-1}=\left(h g^{-1}\right)\left(k^{-1}\right)^{-1} \in Q$, contrary to assumption. Therefore $f_{h}$ and $f_{k}$ are different members of ${ }^{\Phi} G$.

Proposition 5.13. Every l-group is order dense in some p-complete l-group $\boldsymbol{H}$.

Proof. Let $G=G_{0}, G_{\alpha+1}=G_{\alpha}^{p}$ and $G_{\gamma}=\bigcup\left\{G_{\alpha} \mid \alpha<\gamma\right\}$ for limit ordinals $\gamma$. A routine induction reveals that $G \preccurlyeq G_{\alpha}$ for each ordinal $\alpha$. Lemma 5.12 implies that $G_{\alpha}=G_{\alpha+1}$ for some ordinal $\alpha$. Therefore $G_{\alpha}$ is $p$-complete.

THEOREM 5.14. Every l-group $G$ has a p-completion $G^{i p}$ which is unique up to an l-isomorphism over $G$. Every l-monomorphism from $G$ onto a large l-subgroup of a p-complete l-group $K$ has a unique l-monomorphism $\psi^{\wedge}: G^{i p} \rightarrow K$ extending $\psi . G$ and $G^{i p}$ satisfy the same disjunctive formulas.

The most important open question involving $p$-convergence is whether property C5 holds. That is, whether $G^{p}$ is always $p$-complete or whether $G^{i p}=G^{p}$. We shall subsequently provide an affirmative answer for several important classes of $l$ groups, but the general question remains. If, as seems likely, it develops that $G^{p} \neq G^{i p}$ in general, then the most fetching question becomes: which $l$-groups $H$ are of the form $G^{p}$ for some $G \leqslant H$ ?

To motivate the next definition requires an example. Let $G$ be $C(\mathbf{R})$, the continuous real valued functions on $\mathbf{R}$. Let $X$ be any pairwise disjoint subset of $G$ and let $Y$ be $\left\{t_{r} \mid 0 \leqslant r<1\right\}$, where $t_{r} \in G$ is defined by $(s) t_{r}=r$ all $s \in \mathbf{R}$. The theory of lateral completions developed by Conrad and Bernau shows that sets like $X$ may have their suprema adjoined in a consistent fashion. Our contention is that the reason that sets like $X$ have a manageable theory is not exactly disjointness but rather an attribute that may be roughly stated thus: for each $r \in \mathbf{R}$ there is at most one member $x \in X$ such that $(r) \bigvee X=(r) x$. The alert reader will object that $\bigvee X$ is not really a function on $\mathbf{R}$, since the lateral completion of $G$ may not be represented inside $\mathbf{R}^{\mathbf{R}}$ (at least over $G$ ). Nevertheless, the intention of this definition is to express this idea. A subset $Z \subseteq G^{+}$is of type $\mathcal{O}$ if $1=\cap\left\{\left(Z z^{-1} \vee 1\right)^{\perp \perp} \mid z\right.$ $\in Z\}=\cap\left\{\left(z^{-1} Z \vee 1\right)^{\perp \perp} \mid z \in Z\right\}$. The reader should check that $X$ is of type $\mathcal{Y}$ while $Y$ is not.

Proposition 5.15. Every finite subset of $\mathrm{G}^{+}$and every pairwise disjoint subset of $G^{+}$is of type $\mathcal{Y}$.

Proof. If $D$ is a pairwise disjoint set then $\cap\left\{\left(D d^{-1} \vee 1\right)^{\perp \perp} \mid d \in D\right\}=$ $\cap\left\{D^{\perp \perp} \cap d^{\perp} \mid d \in D\right\}=D^{\perp \perp} \cap\left(\cap\left\{d^{\perp} \mid d \in D\right\}\right)=D^{\perp \perp} \cap D^{\perp}=1$. 
Proposition 5.16. Type $\mathcal{O}$ has properties T2 and T3.

Proof. T3 follows directly from Proposition 5.3.

For brevity write $x^{\perp \perp} \cap\left(X x^{-1} \vee 1\right)^{\perp}$ as $Q(x)$. Notice that $x^{-1} Q(x) x=x^{\perp \perp}$ $\cap\left(x^{-1} X \vee 1\right)^{\perp}$. For $X \subseteq G^{+}$let $X^{\prime}=\{x \in X \mid Q(x) \neq 1\}$. We remark in passing that $X \subseteq G^{+}$is type $\mathcal{Y}$ if and only if $X^{\perp \perp}=\bigvee\left\{Q(x) \mid x \in X^{\prime}\right\}=$ $\bigvee\left\{x^{-1} Q(x) x \mid x \in X^{\prime}\right\}$. The point of Proposition 5.18 is that for the purpose of adjoining the supremum of $X$ one may assume $X=X^{\prime}$. The reader should keep in mind that in any l-group, $a \geqslant b$ if and only if $P a \geqslant P b$ for all prime subgroups $P$.

LEMMA 5.17. Suppose $X \subseteq G^{+}$and that $P$ is a prime subgroup such that $Q(x)$ $\nsubseteq P$ for a particular $x \in X$. Then $P x>P y$ for all $y \in X$.

Proof. Consider $1<t \in Q(x)-P$ and arbitrary $y \in X$. Since $t \wedge\left(y x^{-1} \vee 1\right)$ $=1$ and $t \notin P$ it follows that $y x^{-1} \vee 1 \in P$ so that $P y x^{-1}<P$ or $P y<P x$.

Proposition 5.18. Suppose $X \subseteq G^{+}$is of type $\mathcal{Y}$. Then $U(X)=U\left(X^{\prime}\right)$.

Proof. Suppose $y \notin U(X)$, say $y \neq x \in X$. Then $1 \neq\left(x y^{-1} \vee 1\right)^{\perp \perp} \subseteq x^{\perp \perp} \subseteq$ $\bigvee\left\{Q(z) \mid z \in X^{\prime}\right\}$. We may assume the existence of some $t$ such that $1<t \leqslant x y^{-1}$ $\bigvee 1$ with $t \in Q\left(x_{0}\right)$ for some $x_{0} \in X^{\prime}$. Let $P$ be any value of $t$. Since $Q\left(x_{0}\right) \nsubseteq P$ it follows that $P x_{0} \geqslant P z$ for all $z \in X$. Since $P<P t \leqslant P\left(x y^{-1} \vee 1\right)=P x y^{-1} \vee P$ $=P x y^{-1}$ it follows that $P x y^{-1}>P$ or $P x>P y$. Therefore $P x_{0}>P x>P y$, which implies $y \ngtr x_{0} \in X^{\prime}$ so $y \notin U\left(X^{\prime}\right)$.

Lemma 5.19. Suppose $X \subseteq G^{+}$and that $L$ and $M$ are finite subsets of $X^{\prime}$. Then $(\bigvee L)(\bigvee M)^{-1} \in \cap\left\{Q(x)^{\perp} \mid x \in L \cap M\right\}$

Proof. Suppose $x \in L \cap M$. If $(\bigvee L)(\bigvee M)^{-1} \notin Q(x)^{\perp}$ then there is some $v$ such that $1<v \leqslant\left|(\bigvee L)(\bigvee M)^{-1}\right|$ and $v \in Q(x)$. If $P$ is any value of $v$ then $Q(x) \nsubseteq P$ so by Lemma $5.17, P(\bigvee L)=P(\bigvee M)$ and $(\bigvee L)(\bigvee M)^{-1} \in P$. This contradicts $v \notin P$.

Proposition 5.20. Every subset of $G$ of type $\mathcal{O}$ has a supremum in $G^{p}$.

Proof. Let $X \subseteq G^{+}$be of type $\mathcal{Y}$. If $X^{\prime}$ is finite we are done, so suppose $X^{\prime}$ is infinite. For each finite $M \subseteq X^{\prime}$ define $F(M)=\left\{\bigvee L \mid M \subseteq L \subseteq X^{\prime}, L\right.$ finite $\}$ and let $\mathcal{F}$ be the filter generated by all $F(M)$ 's. By Lemma $5.19, F(M) F(M)^{-1} \subseteq$ $\cap\left\{X^{\perp \perp} \cap Q(m)^{\perp} \mid m \in M\right\} ;$ since $\cap\left\{X^{\perp \perp} \cap Q(x)^{\perp} \mid x \in X^{\prime}\right\}=1, \mathscr{F}^{\mathcal{F}} \mathscr{F}^{-1} \rightarrow 1$. An analogous argument, which involves proving the analogues of results 5.17 and 5.19 , shows that $\mathscr{F}^{-1} \mathscr{F} \rightarrow 1$. Therefore $y=[\mathscr{F}] \in G^{p}$. The claim is that $y=\bigvee X^{\prime}$ and therefore $y=\bigvee X$ in $G^{p}$. That $y \geqslant X^{\prime}$ follows from Proposition 1.2. For a particular finite $M \subseteq X^{\prime}, \mathscr{F}(\bigvee M)^{-1} \rightarrow y(\bigvee M)^{-1}$. But $\mathscr{F}(\bigvee M)^{-1}$ contains $F(M)(\bigvee M)^{-1}$, a subset of $\cap\left\{Q(x)^{\perp}\right.$ in $\left.G \mid x \in M\right\}$ by Lemma 5.19. Since $Q(x)^{\perp}$ in $G$ is contained in $Q(x)^{\perp}$ in $G^{p}$, a closed convex $l$-subgroup, it follows that $y(\bigvee M)^{-1} \in \cap\left\{Q(x)^{\perp}\right.$ in $\left.G^{p} \mid x \in M\right\}$. Since $\cap\left\{X^{\perp \perp} \cap Q(x)^{\perp}\right.$ in $\left.G^{p} \mid x \in X^{\prime}\right\}$ $=1, \cap\left\{\left[y(\bigvee M)^{-1}\right]^{\perp \perp} \mid X^{\prime} \supseteq M\right.$ finite $\}=1$ in $G^{p}$. It follows that $y=\bigvee X^{\prime}$, since $\bigvee M \leqslant t \leqslant y$ for all finite $M \subseteq X^{\prime}$ implies $y t^{-1} \in \cap\left[y(\bigvee M)^{-1}\right]^{\perp \perp}$. 
THEOREM 5.21. Every lattice ordered group $G$ has a $\mathcal{Y}$ hull $G_{y}$ which is unique up to a l-isomorphism over $G . G_{\mathscr{y}}$ is laterally complete and is (l-isomorphic to) an $l$-subgroup of $G^{i p} . G$ and $G_{0}$ satisfy the same disjunctive formulas.

A most interesting question is to determine under what circumstances $G_{\mathscr{\vartheta}}=G^{i p}$. The author is willing to conjecture that this is always the case.

If $\mathcal{X}$ in the next corollary is taken to be the type of pairwise disjoint sets then one obtains the existence and uniqueness of the lateral completion, a result due to Bernau [4].

COROllary 5.22. If $\mathcal{X}$ is any type finer than $\mathcal{Y}$ which satisfies $\mathrm{T} 2$ and $\mathrm{T} 3$ then every l-group $G$ has an $\mathcal{X}$ hull $G_{\mathfrak{X}}$ which is unique up to an l-isomorphism over $G$. $G_{\mathfrak{X}}$ is (l-isomorphic to) an l-subgroup of $G^{i p}$. $G$ and $G_{\mathfrak{X}}$ satisfy the same disjunctive formulas.

COROLlaRy 5.23. For every l-group $G, G^{i p}$ is sup $\mathcal{Y}$-complete, hence laterally complete.

$G_{0 y}$ is strictly larger than the lateral completion of $G$ in general. For example, let $\Gamma$ be all finite sequences from $N=\{1,2,3, \ldots\}$ including the sequence $\lambda$ of zero length. Order $\Gamma$ by declaring $\alpha \geqslant \beta$ whenever $\alpha$ is an initial segment of $\beta$. Let $V$ designate the group of all functions from $\Gamma$ into $R$ ordered by declaring that $f \geqslant g$ if $(\beta) f<(\beta) g$ implies the existence of some $\alpha>\beta$ such that $(\alpha) f>(\alpha) g$. This construction of $V$ from $\Gamma$ is an instance of a general construction of central importance to the theory of abelian $l$-groups [9]. Let $G$ be those $g \in V$ which satisfy the following condition. For each $\alpha \in \Gamma$ there is some $k \in N$ such that for every $m>k,(\alpha m) g=0$ and $(\alpha m i) g=(\alpha i) g$ for all $i \in N$.

$G$ is laterally complete. To see this let $D$ be a pairwise disjoint subset of $G$ and let $x$ be the supremum of $D$ in $V$. The claim is that $x$ is in $G$. Consider an arbitrary $\alpha \in \Gamma$. Then there are at most finitely many elements $d \in D$ such that $(\alpha n) d \neq 0$ for some $n \in N$. (In fact, if $(\alpha n) d \neq 0$ for some $n$ and if $k \in N$ satisfies $(\alpha m i) d=$ ( $\alpha i) d$ for all $i$ and all $m>k$ then there can be at most $k$ other members $d^{\prime} \in D$ such that $(\alpha n) d^{\prime} \neq 0$ for some $n$.) Label these elements $d_{1}, d_{2}, \ldots, d_{p}$. For each $d_{j}$ let $k_{j} \in N$ satisfy $(\alpha m) d_{j}=0$ and $(\alpha m i) d_{j}=(\alpha i) d_{j}$ for all $i$ and all $m>k_{j}$. Let $k=\bigvee k_{j}$. Then $(\alpha m) x=0$ and $(\alpha m i) x=(\alpha i) x$ for all $i$ and all $m>k$. Therefore $x \in G$.

$G$ is not sup $\mathcal{Y}$-complete. To demonstrate this we need only pick out a type $\mathcal{Y}$ subset of $G$ whose supremum in $V$ is not in $G$. To that end let $g_{n}$ be any member of $G$ such that $(\lambda) g_{n}=0$ and $(k) g_{n}=0$ if $k \neq n$ and $(n) g_{n}=1$. The collection of $g_{n}$ 's is not a disjoint set but is type $\mathcal{Y}$. Furthermore, its supremum is not in $G$.

The reader may verify that $G_{\mathscr{y}}$ consists of those $v \in V$ which satisfy the following condition. For each $\alpha \in \Gamma$ there is some $\beta \leqslant \alpha$ and some $k \in N$ such that $(\beta m) v=0$ and $(\beta m i) v=(\beta i) v$ for all $i$ and all $m>k$.

Proposition 5.20 makes it possible to prove that a convex $l$-subgroup is $p$-closed if and only if it is order closed.

Proposition 5.24. If $C$ is a convex l-subgroup of $G$ then $\operatorname{cl}(C)=\operatorname{ocl}(C)$. 
Proof. Suppose $1<h=\bigvee S$ where $S=\left\{c \in C^{+} \mid c \leqslant h\right\}$. If $S$ can be shown to be type $\mathcal{Y}$, then the proof of Proposition 5.20 will provide a filter $\mathscr{F}$ on $C$ such that $\mathscr{F} \rightarrow h$, proving the present proposition. Suppose $S$ is not of type $\mathcal{Y}$, that is, suppose $1<c \in S$ satisfies $c \in\left[s^{\perp \perp} \cap\left(S s^{-1} \vee 1\right)^{\perp}\right]^{\perp}$ for all $s \in S$. If it can be proven that $S c \subseteq S$ we will be done, since this condition contradicts $\bigvee S=h$. To that end suppose there is $s_{0} \in S$ with $s_{0} c \vee h>h$. Let $s_{0} c \wedge h=s_{1} \in S$. Since $\left(s_{0} c \vee h\right) h^{-1} \in c^{\perp \perp}$ we may find $t>1$ such that $t \leqslant c \wedge\left(s_{0} c \vee h\right) h^{-1}$. It follows that $t \leqslant s_{1}$. The proof is completed by showing that $t \in\left(S s_{1}^{-1} \vee 1\right)^{\perp}$, which contradicts $c \in\left[s_{1}^{\perp \perp} \cap\left(S s_{1}^{-1} \vee 1\right)^{\perp}\right]^{\perp}$. This can be accomplished by showing that for any prime subgroup $P$ and any $s \in S, P t \wedge P\left(s s_{1}^{-1} \vee 1\right)=P$. Therefore assume that $P$ is a prime subgroup with $P<P t$. Then $P t \leqslant P\left(s_{0} c \vee h\right) h^{-1}$ implies $P s_{0} c>P h$ so that $P s_{1}=P s_{0} c \wedge P h=P h$. For any $s \in S, P s \leqslant P h=P s$ so $P\left(s s_{1}^{-1} \vee 1\right)=P$. This completes the proof.

COROLlaRY 5.25. The p-closure operator is idempotent on convex l-subgroups.

The remainder of this section consists of descriptive results about $G^{p}, G^{i p}$ and, hence, about $G_{9 y}$. An element $1<b \in G$ is basic if the convex $l$-subgroup generated by $b$ is totally ordered. $b \in G^{+}$is basic if and only if $b>1$ and $b^{\perp}$ is prime [9]. An $l$-group $G$ has $a$ basis if each $1<g \in G$ exceeds a basic element. $G$ has a finite basis if there is a finite maximal pairwise disjoint set of basic elements. The next result uses only the methods and terminology of $\S 1$ and could have been proven there.

Proposition 5.26. Suppose $G$ is an l-subgroup of the Hausdorff lc-group $(H, \Rightarrow)$. If $b$ is basic in $G$ then $b$ is basic in $\operatorname{cl}(G)$. If $G$ has a basis and if $G$ is order dense in $\operatorname{cl}(G)$ then $\operatorname{cl}(G)$ has a basis.

Proof. Suppose $b$ is basic in $G$ and let $P=b^{\perp}$ in $G, Q=b^{\perp}$ in $\operatorname{cl}(G)$. Now $\operatorname{cl}(P) \subseteq Q$, for if $\mathcal{F}$ is a filter such that $P \in \mathcal{F} \rightarrow h \in H^{+}$then $\{1\}=(P \vee 1) \wedge$ $b \in(\mathscr{F} \vee 1) \wedge b \rightarrow(h \vee 1) \wedge b=h \wedge b$, implying $h \wedge b=1$. Since $\operatorname{cl}(P)$ is a prime subgroup of $\operatorname{cl}(G), Q$ must be prime in $\operatorname{cl}(G)$ so $b$ is basic in $\operatorname{cl}(G)$.

In the next proposition $\left(B, \leqslant, \vee, \wedge,{ }^{\perp}, 0,1\right)$ is a Boolean algebra. An element $b \in B$ is an atom if $b \neq 0$ and if $0 \leqslant a \leqslant b$ implies $a=0$ or $a=b$. $B$ is atomic if every nonzero element exceeds an atom. $F \subseteq B$ is a filter if $b \geqslant f \in F$ implies $b \in F$ and if $f_{1}, f_{2} \in F$ implies $f_{1} \wedge f_{2} \in F$. The inclusion order is assumed on filters. The following result is jointly due to Otis Kenny and the author.

Proposition 5.27. $A$ Boolean algebra $B$ admits a minimal filter $F$ such that $\wedge F=0$ if and only if $B$ is atomic and $F=\left\{b \in B \mid b \geqslant a_{1}^{\perp} \wedge a_{2}^{\perp} \wedge \cdots \wedge a_{n}^{\perp}, a_{i}\right.$ atoms $\}$.

Proof. Suppose $M$ is any filter such that $\wedge M=0$ and suppose $a$ is an atom. There must be some $m \in M$ such that $a \nless m$ so that $a \wedge m=0$ and $m \leqslant a^{\perp}$, putting $a^{\perp}$ in $M$. Therefore $F \subseteq M$. Since $\bigvee\{a \mid a$ an atom $\}=1, \wedge F=0$. Now suppose that $B$ has a coarsest filter $F$ such that $\wedge F=0$, yet there is some 
$0 \neq b \in B$ exceeding no atom. Choose $f \in F$ such that $f \neq b$. Notice that $1 \neq f \vee$ $b^{\perp} \in F$ so that $f^{\perp} \wedge b \neq 0$ and $f^{\perp} \wedge b$ exceeds no atom. Let $m_{0}$ be $f^{\perp} \wedge b$, let $m_{\alpha+1}$ satisfy $0<m_{\alpha+1}<m_{\alpha}$ and let $m_{\gamma}$ satisfy $0<m_{\gamma}<m_{\alpha}$ for all $\alpha<\gamma$. Continue this process until at some limit stage no choice of $m_{\gamma}$ is possible. Let $M=\left\{b \in B \mid b \geqslant m_{\alpha}\right.$ some $\left.\alpha\right\}$, a filter on $B$ such that $\wedge M=0$. Since $M \supseteq F$ there must be some $\alpha$ such that $f \vee b^{\perp} \geqslant m_{\alpha}$. But this contradicts $\left(f \vee b^{\perp}\right)^{\perp}=$ $m_{0} \geqslant m_{\alpha}>0$.

The connection between the last two results is provided by the observation that a polar $P$ is a prime convex $l$-subgroup if and only if $P^{\perp}$ is an atom in the Boolean algebra of polars.

Proposition 5.28. Polar convergence is topological if and only if $G$ has a basis. It is discrete if and only if $G$ has a finite basis.

Proposition 5.29. If $G$ has $a$ basis then $G^{p p}=G^{p}$ and the extension by regularity of $\rightarrow$ on $G$ coincides with $\rightarrow$ on $G^{p}$.

Proof. In this case $G^{p}$ is just the completion of $G$ with respect to the two-sided uniformity of the $p$-topology on $G$ and the canonical uniformity on $G^{p}$ is just the two-sided uniformity of the $p$-topology on $G^{p}$. Hence $G^{p}$ is $p$-complete. The statement about extension by regularity is also a standard feature of uniformities.

TheOREM 5.30. Suppose $G$ has a basis. For $G \leqslant H$ the following are equivalent.

(a) $H$ is the p-completion of $G$.

(b) $H$ is p-complete and every p-continuous l-homomorphism from $G$ into a p-complete l-group $M$ can be uniquely extended to a p-continuous l-homomorphism $\psi^{\wedge}: H \rightarrow M$.

(c) $G \leqslant_{p} H$, and $H \leqslant_{p} M$ implies $H=M$.

(d) $G \leqslant_{p} H$, and $H \leqslant M$ together with $G \leqslant_{p} H$ imply $H=M$.

Proposition 5.31. In a strongly projectable l-group $G$ every $1<x \in G^{p}$ is $a$ supremum of a pairwise disjoint subset of $\mathrm{G}^{+}$.

Proof. For each polar $P$ of $G$ let $\pi P: G \rightarrow P$ be the projection $l$-epimorphism such that $\pi P$ is the identity of $P$ and $g=(g \pi P)\left(g \pi P^{\perp}\right)$ for all $g \in G$ and all polars $P$. Consider an arbitrary $1<x \in G^{p}$ and a filter $\mathcal{F}$ such that $G^{+} \in \mathcal{F} \rightarrow x$. Notice that for any $F \in \mathcal{F}$ with $F \subseteq G^{+}$and for any polar $Q$ of $G$ containing $F F^{-1}, f_{1} \pi Q^{\perp}=f_{2} \pi Q^{\perp}$ for all $f_{1}, f_{2} \in F$. Let $\left\{F_{\alpha} \mid \alpha<\kappa\right\}$ be a well-ordering of those sets in $\mathcal{F}$ which are subsets of $G^{+}$. Define $d_{0}=f \pi Q_{0}$ where $f \in F_{0}$ and $Q_{0}=\left(F_{0} F_{0}^{-1}\right)^{\perp}$. Having defined $d_{\alpha}$ and $Q_{\alpha}$ for all $\alpha<\beta$ let $d_{\beta}=f \pi Q_{\beta}$ where $f \in F_{\beta}$ and $Q_{\beta}=\cap\left\{\left(F_{\alpha} F_{\alpha}^{-1}\right)^{\perp \perp} \mid \alpha<\beta\right\} \cap\left(F_{\beta} F_{\beta}^{-1}\right)^{\perp}$. A simple induction shows that $d_{\alpha} \wedge d_{\beta}=1$ for $\alpha<\beta<\kappa$. We claim $x=\bigvee d_{\alpha}$. It is clear that $x>d_{\alpha}$ for each $\alpha$ since for every $f \in F_{\alpha}, f \geqslant f \pi Q_{\alpha}=d_{\alpha}$. Now observe that for each $\alpha$, $\mathscr{F} d_{\alpha}^{-1} \rightarrow x d_{\alpha}^{-1} \geqslant 1$ and $\mathscr{F} d_{\alpha}^{-1}$ contains $F_{\alpha} d_{\alpha}^{-1} \subseteq Q_{\alpha}^{\perp}$. Therefore $x d_{\alpha}^{-1} \in \operatorname{cl}\left(Q_{\alpha}^{\perp}\right) \subseteq$ $Q_{\alpha}^{\perp}$ in $G^{p}$. It follows from $\cap\left\{\left(F F^{-1}\right)^{\perp \perp} \mid F \in \mathscr{F}\right\}=1$ that $\cap\left\{Q_{\alpha}^{\perp} \mid \alpha<\kappa\right\}=1$ in $G$ and that $\cap\left\{Q_{\alpha}^{\perp}\right.$ in $\left.G^{p} \mid \alpha<\kappa\right\}=1$. But then $\bigvee d_{\alpha}=x$, for if $d_{\alpha}<t<x$ for all $\alpha$, then $x t^{-1} \in \cap Q_{\alpha}^{\perp}=1$. 
Proposition 5.32. If $G$ is strongly projectable then so is $G^{p}$.

Proof. Suppose $1<x \in G^{p}$ and $Q$ is a polar of $G^{p}$. Bruising the notation of the previous result a bit, let $\pi Q: G \rightarrow Q \cap G$ and $\pi Q^{\perp}: G \rightarrow Q^{\perp} \cap G$ be the projection maps. Let $D$ be a pairwise disjoint subset of $G^{+}$such that $x=\bigvee D$. Let $A=D \pi Q$ and $B=D \pi Q^{\perp}$, pairwise disjoint subsets of $Q \cap G$ and $Q^{\perp} \cap G$ respectively. By Proposition 5.20 there are elements $a$ and $b$ in $G^{p}$ such that $a=\bigvee A$ and $b=\bigvee B$. Since $Q$ and $Q^{\perp}$ are order closed, $a \in Q$ and $b \in Q^{\perp}$. Finally, $x \geqslant A$ and $x \geqslant B$ implies $x \geqslant a \vee b=a b$, and $a b \geqslant D$ implies $a b \geqslant x$, so $a b=x$.

Proposition 5.33. If $G$ is strongly projectable then $G^{p p}=G^{p}$.

Proof. Consider $1<x \in G^{p p}$. Since $G^{p}$ is strongly projectable there is a pairwise disjoint set $X \subseteq G^{p+}$ such that $x=\bigvee X$. For each $1<d \in X$ there is a pairwise disjoint set $D_{d} \subseteq G^{+}$such that $d=\bigvee D_{d}$. Since $D=\cup\left\{D_{d} \mid d \in X\right\}$ is a pairwise disjoint subset of $G$, Proposition 5.20 guarantees $x=\bigvee D \in G^{p}$.

LEMMA 5.34. If $G$ is strongly projectable then for any polar $P$ of $G, \operatorname{cl}(P)=P^{\perp \perp}$ in $G^{p}$.

Proof. Consider $1<x \in P^{\perp \perp}$ and let $D$ be a disjoint subset of $G^{+}$such that $\bigvee D=x$. Then $D \subseteq P^{\perp \perp} \cap G=P$. For each finite $M \subseteq D$ let $F(M)=$ $\{\bigvee L \mid M \subseteq L \subseteq D$ and $L$ finite $\}$ and let $\mathscr{F}$ be the filter generated by the $F(M)$ 's. An argument like the proof of Proposition 5.24 shows that $\mathscr{F} \rightarrow x$. Therefore $x \in \operatorname{cl}(P)$.

Proposition 5.35. If $G$ is archimedean so is $G^{p}$.

Proof. Suppose $1<a<b \in G^{p}$. We wish to show $a^{n} \nless b$ for some $n$. Let $\mathscr{F}$ be a filter such that $G \in \mathcal{F} \rightarrow b$ and such that for all $F \in \mathcal{F}$ there is some $f \in F$ with $f>a$. We may assume that $a \in G$ and that $a \in\left(F F^{-1}\right)^{\perp}$ for some $F \in \mathcal{F}$ such that $F \subseteq G$. Fix $f \in F$ such that $f \geqslant a$. Since $G$ is archimedean there is an integer $n$ such that $a^{n} \nless f$. We claim that actually $a^{n} \nless b$. To establish this claim it is enough to show that for any filter $\mathfrak{N}$ such that $G \in \mathfrak{N} \rightarrow a^{n}$ there is some $M \in \mathfrak{N}$ such that no member of $F$ exceeds any member of $M$. To that end consider an arbitrary $\mathfrak{N}$ with $G \in \mathfrak{N} \rightarrow a^{n}$. Since $\Re a^{-n} \rightarrow 1$, there must be some $M \in \mathscr{N}$ such that $1<\left(a^{n} \vee f\right) f^{-1}=a^{n} f^{-1} \vee 1 \notin\left(M a^{-n}\right)^{\perp \perp}$. That is, there exists $t \in\left(M a^{-n}\right)^{\perp}$ such that $1<t<a^{n} f^{-1} \vee 1$. Let $P$ be any value of t. Then $P<P t<P a^{n} f^{-1} \vee P=P a^{n} f^{-1}$ so $P a^{n}>P f$. Since $F F^{-1} \subseteq a^{\perp} \subseteq$ $\left(a^{n} f^{-1} \vee 1\right)^{\perp} \subseteq t^{\perp}$ and since $t \notin P$ it follows that $F F^{-1} \subseteq P$ and $P f=P f_{0}$ for all $f_{0} \in F$. Finally, $\left(M a^{-n}\right) \subseteq t^{\perp}$ implies $P m=P a^{n}$ for all $m \in M$. Therefore for any $m \in M$ and $f_{0} \in F, P m=P a^{n}>P f=P f_{0}$, so $m \nless f_{0}$.

THEOREM 5.36. Suppose $G$ is archimedean or strongly projectable and suppose $G<H$. Then the following are equivalent.

(a) $H=G_{y}$.

(b) $H$ is the lateral completion of $G$. 
(c) $H$ is the orthocompletion of $G$.

(d) $H$ is the $p$-completion of $G$.

(e) $H$ is p-complete and every p-continuous l-homomorphism $\psi$ mapping $G$ into a p-complete l-group $M$ can be uniquely extended to a p-complete l-homomorphism $\psi^{\wedge}$ : $H \rightarrow M$.

(f) $\mathrm{g} \leqslant_{p} H$, and $H \leqslant_{p} M$ implies $H=M$.

(g) $G \leqslant_{p} H$, and $H \leqslant M$ together with $G \leqslant_{p} M$ imply $H=M$.

Proof. Bernau [3] has shown that every laterally complete archimedean $l$-group is strongly projectable. Therefore, by Proposition 5.24, an archimedean $l$-group is $p$-complete if and only if it is laterally complete.

Not all $p$-complete $l$-groups are strongly projectable. Let $\Gamma$ be as in the example following Corollary 5.23 and let $G=\left\{g \in V(\Gamma, \mathbf{R}) \mid\left(a_{n}\right) g=\left(a_{m}\right) g\right.$ all $\left.n, m\right\}$. Then $G$ is $p$-complete but not projectable. Notice that $G$ has a basis.

Proposition 5.37. If $G$ is large in $H$ then $G^{p} \leqslant H^{p}$. If, for some collection $\left\{H_{\lambda} \mid \lambda \in \Lambda\right\}$ of l-groups, $\Sigma H_{\lambda} \leqslant G \leqslant \Pi H_{\lambda}$ then $\Sigma H_{\lambda}^{p} \leqslant G^{p} \leqslant \Pi H_{\lambda}^{p}$.

Proof. Corollary 5.8 and Proposition 2.25 establish the first claim. If $\Sigma H_{\lambda}<G$ $\leqslant \Pi H_{\lambda}$ then the Boolean algebra of polars of $G$ is the product of the individual polar Boolean algebras on the $H_{\lambda}$ 's. Therefore polar convergence on $G$ is the product of the polar convergences on the $H_{\lambda}$ 's. $\mathrm{C} 7$ therefore holds and Proposition 2.32 proves the second statement.

Polar convergence does not have property C6. In particular, the conclusion of Proposition 2.31 is invalid for polar convergence. As an example of this phenomenon let $\Gamma$ be a binary tree growing downwards. More precisely, let $\Gamma$ be the set of all finite strings of 0 's and 1's including the empty string $\lambda$. For $\alpha, \beta \in \Gamma$ define $\alpha \geqslant \beta$ if $\alpha$ is an initial segment of $\beta$. Let $G=\{g \in V(\Gamma, \mathbf{R}) \mid g$ has finite support $\}$. A good deal of cogitation is required, but one may show $G^{p}=\{g \in V(\Gamma, \mathbf{R}) \mid$ the support of $g$ has holes $\}$. A set $\Delta \subseteq \Gamma$ has holes if for every $\delta \in \Delta$ there is some $\alpha<\delta$ such that $\beta \leqslant \alpha$ implies $\beta \notin \Delta$. Let $P=\left\{g \in G \mid\left(0^{n}\right) g=0\right.$ for all $\left.n\right\}$ and $Q=\left\{g \in G^{p} \mid\left(0^{n}\right) g=0\right.$ all $\left.n\right\}$. Then $P(Q)$ is order and $p$-closed in $G\left(G^{p}\right)$ and in fact $\operatorname{ocl}(P)=\operatorname{cl}(P)=Q$ in $G^{p}$. However $G / P$ is $\left\{g \in V\left(N^{-}, \mathbf{R}\right) \mid g\right.$ has finite support $\}$ while $G^{p} / Q$ is $V\left(N^{-}, \mathbf{R}\right)$, where $N^{-}=\{\ldots,-2,-1\}$. This is in spite of the fact that $(G / P)^{p}=G / P$, since the latter has a finite basis.

Suppose $G$ is a representable $l$-group and $\left\{P_{\lambda} \mid \lambda \in \Lambda\right\}$ a set of normal primes intersecting to 1 . For each $\lambda$ let $T_{\lambda}$ be the totally ordered group $G / P_{\lambda}$. We may think of $G$ as a subdirect product of the $T_{\lambda}$ 's. With each polar $P$ associate $S(P)=\{\lambda \in \Lambda \mid(\lambda) g \neq 0$ for some $g \in P\}$. Notice that $S\left(P_{1} \cap P_{2}\right)=S\left(P_{1}\right) \cap$ $S\left(P_{2}\right)$ and $S\left(P_{1} \vee P_{2}\right)=S\left(P_{1}\right) \cup S\left(P_{2}\right)$, so that $\{S(P) \mid P$ a polar of $G\}$ is a Boolean algebra of subsets of $\Lambda$. Let $H$ be the collection of all $h \in \Pi T_{\lambda}$ satisfying the following condition. There is a filter $\mathscr{P}$ of polars of $G$ such that $\cap \mathscr{P}=1$ and for every $P \in \mathcal{P}$ there is some $g \in G$ with $(\lambda) g=(\lambda) h$ for all $\lambda \in S\left(P^{\perp}\right) . H$ is an $l$-subgroup of $\Pi T_{\lambda}$ containing $G$. Let $N$ be the collection of all $n \in H$ satisfying the following condition. There is a filter $\mathscr{P}$ of polars of $G$ such that $\cap \mathscr{P}=1$ and 
$(\lambda) n=1$ for all $\lambda \in \cup\left\{S\left(P^{\perp}\right) \mid P \in \mathcal{P}\right\} . N$ is an $l$-ideal of $H$ such that $N \cap G=$ 1 .

Proposition 5.38. Let $G,\left\{P_{\lambda}\right\},\left\{T_{\lambda}\right\}, H$ and $N$ be as above. Then the projection map $\theta: G \rightarrow H / N$ can be extended to an l-isomorphism $\theta^{\wedge}$ from $G^{p}$ onto $H / N$.

Proof. Given $[\mathscr{P}] \in G^{p}$ let $\mathscr{P}$ be $\left\{\left(F F^{-1}\right)^{\perp \perp} \mid F \in \mathscr{F}\right\}$. Note that $\cap \mathscr{P}=1$, and that $f_{1}, f_{2} \in F \in \mathscr{F}$ implies $(\lambda) f_{1}=(\lambda) f_{2}$ for $\lambda \in S\left(\left(F F^{-1}\right)^{\perp}\right)$. Define $h \in H$ as follows: if $\lambda \in S\left(\left(F F^{-1}\right)^{\perp}\right)$ for some $F \in \mathscr{F}$ let $(\lambda) h=(\lambda) f$ for any $f \in F$; otherwise, let $(\lambda) h=1$. Finally, let $[\mathcal{F}] \theta^{\wedge}=N h$. The reader may verify that $\theta^{\wedge}$ is an $l$-isomorphism extending $\theta$.

This result is unsatisfactory for representing $G^{i p}$, however, since the conclusion of the theorem leaves in doubt how best to represent $G^{p}$. In particular, one might ask: when can an $l$-monomorphism $\theta$ from $G$ onto a subdirect product of totally ordered groups $\Pi T_{\lambda}$ be extended to an $l$-monomorphism $\theta^{\wedge}: G^{i p} \rightarrow \Pi T_{\lambda}$ ? A minimal requirement is that the $P_{\lambda}$ 's be $p$-closed; hence $G$ must be completely distributive. The example following Proposition 5.37 shows that even in this case $T_{\lambda}=G / P_{\lambda} \neq\left(G^{p} / \operatorname{cl}\left(P_{\lambda}\right)\right)$. The alert reader will have noticed that in this example $G^{p} / \operatorname{cl}\left(P_{\lambda}\right)=\left(G / P_{\lambda}\right)^{\wedge}$. It can be shown in general that if the $P_{\lambda}$ 's are order closed then $G^{i p} \leqslant \Pi\left(T_{\lambda}^{\wedge}\right)$. But this is simply a representation of $G^{\alpha}$, the completion of $G$ with respect to the uniformity of the $\alpha$ topology, a subject which will be taken up in a forthcoming paper. Similar remarks apply to the analogous of Proposition 5.38 resulting from the other classical $l$-group representation theorems.

\section{REFERENCES}

1. R. N. Ball, Topological lattice ordered groups, Pacific J. Math. (to appear).

2. B. Banaschewski, Über die Vervollständigung geordneter Gruppen, Math. Nachr. 16 (1957), 52-71.

3. S. J. Bernau, Lateral and Dedekind completion of archimedean lattice groups, J. London Math. Soc. 12 (1976), 320-322.

4. _ The lateral completion of an arbitrary lattice group, J. Austral. Math. Soc. Ser. A 19 (1975), 263-289.

5. _, Varieties of lattice groups are closed under L-completion, Symposia Math. 21 (1977), 349-355.

6. R. D. Bleier and P. F. Conrad, $a^{*}$-closures of lattice-ordered groups, Trans. Amer. Math. Soc. 209 (1975), 367-387.

7. Bourbaki, Elements of mathematics, General Topology. I, Addison-Wesley, Reading, Mass., 1966.

8. R. D. Byrd and J. T. Lloyd, Closed subgroups and complete distributivity in lattice ordered groups, Math. Z. 101 (1967), 123-130.

9. P. F. Conrad, Lattice-ordered groups, Lecture notes, Tulane University, 1970.

10. The topological completion and the linearly compact hull of an abelian l-group, Proc. London Math. Soc. 28 (1974), 457-481.

11. P. F. Conrad and D. McAlister, The completion of a lattice ordered group, J. Austral. Math. Soc. 9 (1969), 182-208.

12. R. E. DeMarr, Order convergence and topological convergence, Proc. Amer. Math. Soc. 16 (1965), 588-590.

13. C. J. Everett and S. Ulam, On ordered groups, Trans. Amer. Math. Soc. 57 (1945), 208-216.

14. W. C. Holland, Extensions of ordered groups and sequence completion, Trans. Amer. Math. Soc. 107 (1963), 71-82.

15. G. O. Kenny, The completion of an abelian l-group, Canad. J. Math. 27 (1975), 980-985.

16. D. C. Kent, On the order topology in a lattice, Illinois J. Math. 10 (1966), 90-96.

17. D. C. Kent and G. D. Richardson, Regular completions of Cauchy spaces, Pacific J. Math. 51 (1974), 483-490. 
18. R. L. Madell, Embeddings of topological lattice ordered groups, Trans. Amer. Math. Soc. 146 (1969), 447-455.

19. F. Papangelou, Order convergence and topological completion of commutative lattice-groups, Math. Ann. 155 (1964), 81-107.

20. Some considerations on convergence in abelian lattice groups, Pacific J. Math. 15 (1965), 1347-1364.

21. R. H. Redfield, Ordering uniform completions of partially ordered sets, Canad. J. Math. 26 (1974), 644-664.

22. E. E. Reed, Completions of uniform convergence spaces, Math. Ann. 194 (1971), 83-108.

Department of Mathematics, Boise State University, Boise, Idaho 83725 